\title{
The Hamburg/RASS Catalogue of optical identifications
}

\section{Northern high-galactic latitude ROSAT Bright Source Catalogue X-ray sources}

\author{
F.-J. Zickgraf ${ }^{1}$, D. Engels ${ }^{1}$, H.-J. Hagen ${ }^{1}$, D. Reimers ${ }^{1}$, and W. Voges ${ }^{2}$ \\ 1 Hamburger Sternwarte, Gojenbergsweg 112, 21029 Hamburg, Germany \\ 2 Max-Planck-Institut für extraterrestrische Physik, Postfach 1312, 85741 Garching, Germany
}

Received 2 August 2002 / Accepted 6 May 2003

\begin{abstract}
We present the Hamburg/RASS Catalogue (HRC) of optical identifications of X-ray sources at high-galactic latitude. The HRC includes all X-ray sources from the ROSAT Bright Source Catalogue (RASS-BSC) with galactic latitude $|b| \geq 30^{\circ}$ and declination $\delta \geq 0^{\circ}$. In this part of the sky covering $\sim 10000 \mathrm{deg}^{2}$ the RASS-BSC contains $5341 \mathrm{X}$-ray sources. For the optical identification we used blue Schmidt prism and direct plates taken for the northern hemisphere Hamburg Quasar Survey (HQS) which are now available in digitized form. The limiting magnitudes are 18.5 and 20, respectively. For $82 \%$ of the selected RASS-BSC an identification could be given. For the rest either no counterpart was visible in the error circle or a plausible identification was not possible. With $\sim 42 \%$ AGN represent the largest group of X-ray emitters, $\sim 31 \%$ have a stellar counterpart, whereas galaxies and cluster of galaxies comprise only $\sim 4 \%$ and $\sim 5 \%$, respectively. In $\sim 3 \%$ of the RASS-BSC sources no object was visible on our blue direct plates within $40^{\prime \prime}$ around the X-ray source position. The catalogue is used as a source for the selection of (nearly) complete samples of the various classes of X-ray emitters.
\end{abstract}

Key words. surveys - X-rays: general - X-rays: galaxies - X-rays: stars - galaxies: active - galaxies: clusters: general

\section{Introduction}

The ROSAT All-Sky Survey (RASS) was performed in 1990/91 within the first six months of the ROSAT X-ray satellite mission (Trümper 1983). It was the first all-sky survey in the soft $\mathrm{X}$-ray range from $0.07 \mathrm{keV}$ to $2.4 \mathrm{keV}$ with an imaging telescope (Aschenbach 1988). The results of the survey, photon data as well as source catalogues, have been made available to the community. In total nearly $125000 \mathrm{X}$-ray sources were detected and published in two catalogues. The ROSAT Bright Source Catalogue (RASS-BSC), contains 18811 ROSAT allsky survey sources with count-rates $\geq 0.05 \mathrm{cts} \mathrm{s}^{-1}$ in the $0.1-$ $2.4 \mathrm{keV}$ energy range (Voges et al. 1999). The ROSAT Faint Source Catalogue (RASS-FSC) comprises 105924 X-ray sources with count rates below $0.05 \mathrm{cts} \mathrm{s}^{-1}$ (Voges et al. 2000).

For follow-up studies the nature of the sources must be known in order to select objects of the class to be studied from the RASS source list. The ROSAT data alone usually do not allow one to identify the nature of an X-ray source. Additional information, in particular from optical observations, is necessary. In the past decade numerous programs for the optical identification of RASS X-ray sources have been performed. Many of these programs aimed at the identification of objects

Send offprint requests to: F.-J. Zickgraf, e-mail: fzickgraf@hs. uni-hamburg.de belonging to certain classes of X-ray emitters by applying preselection criteria, like X-ray count rates, hardness ratios and source extension. Other programs aimed at a statistical identification of the RASS without preselection on the basis of X-ray properties. Two studies of this type reaching the RASS detection limit are the ROSAT North Ecliptic Pole Survey (Henry et al. 2001; Voges et al. 2001) and the LSW-INAOE-MPE project (Zickgraf et al. 1997; Appenzeller et al. 1998; Krautter et al. 1999). In these studies the sky area and the number of sources were relatively small, $\simeq 1-2 \%$ of the sky and $\$ 1 \%$ of the RASS sources, respectively. An investigation covering a large area of sky is the ROSAT Bright Survey (RBS) of $\sim 2000$ X-ray bright RASS sources with count rates of $>0.2 \mathrm{cts} \mathrm{s}^{-1}$ (Fischer et al. 1998; Schwope et al. 2000).

The nature of the vast majority of RASS sources is still unknown. The potential of the RASS lying in the all-sky coverage with high sensitivity has still not been fully exploited. This is particularly important in searching for rare objects which require one to survey large areas of the sky. The optical identification process can be made significantly more efficient if a preselection of the possible optical counterparts is available. The Hamburg/RASS optical identification project aims at the identification of all RASS sources in the northern extragalactic sky as a basis for detailed follow-up studies. This goal can be reached by using Schmidt prism plates covering large 
areas of sky. In this way we have finished the identification of all RASS-BSC sources with $\delta \geq 0^{\circ}$ and galactic latitudes $|b| \geq 30^{\circ}$. In this region of the sky covering $10313 \mathrm{deg}^{2}$ the BSC contains 5341 X-ray sources.

A first version of the Hamburg/RASS Catalogue of optical identifications (HRC) containing identifications of 4190 RASS-BSC sources in the sky region defined above plus additional sources in the galactic latitude range $20^{\circ} \leq|b| \leq$ $30^{\circ}$ was published by Bade et al. (1998a) (Paper I hereafter). Here we present the full catalogue of the complete sample of 5341 RASS-BSC sources. The catalogue itself is available electronically ${ }^{1}$. In Sect. 2 the identification technique is summarized. Identification criteria are discussed in Sect. 3. The catalogue is described in Sect. 4. A statistical analysis is given in Sect. 5 and finally the results are summarized in Sect. 6.

\section{Identification technique}

The identification of the RASS-BSC sources was performed in two steps. First, the optical sources in the error circles of the RASS sources were classified. Then the optical and the X-ray data were combined to assign the most likely counterpart to the $\mathrm{X}$-ray source.

The optical classification is based on 567 blue Schmidt prism plates and a set of blue direct plates collected during the Hamburg Quasar Survey (HQS) covering the northern high galactic latitude sky with $|b|>20^{\circ}$ (Hagen et al. 1995). Because of the strong increase in the overlap rate of spectra towards low galactic latitude we restricted the RASS identification to galactic latitude $|b|>30^{\circ}$. The average limiting magnitudes are $B_{\text {lim }, \mathrm{p}}=18.5$ for the prism plates and $B_{\text {lim, }}=20$ for the direct plates. The identification technique used for the first version of the HRC is described in detail in Paper I. Although since then some modifications of the procedure have been implemented, the general identification process for the remaining $\sim 1200$ sources was the same as for the first part of the HRC with the exception of the scanning procedure. In particular, we have now at our disposal digitized high resolution scans of one of the two Schmidt prism plates obtained for each field. The identification procedure for the new sources was as follows. We first searched on the digitized direct plates for all possible counterparts in the X-ray error circle. A search radius of $60^{\prime \prime}$ was adopted. Note that this is significantly larger then the nominal ROSAT $3 \sigma$ error circle which is $\approx 40^{\prime \prime}$ (Voges et al. 1999). For a few Schmidt prism fields no direct plates were available. In these cases we used the Digitized Sky Survey instead. Then spectra were extracted from the data base of the digitized spectral plates at the positions of the objects instead of scanning the plates individually as described in Paper I. Using these spectra the objects were classified interactively. Object classes used for the classification are listed in Table 1. In Sect. 3 more details on classification criteria are given. Finally, all objects were entered into the catalogue and the most likely counterparts to the $\mathrm{X}$-ray sources were identified by a flag. For the source identification the codes and object categories listed in Table 2 were used. Object classes, ID codes and object categories are the

\footnotetext{
${ }^{1}$ http://www.hs.uni-hamburg.de/hrc.html
}

same as in Paper I. We then created the final catalogue by extracting the 4190 entries from the first version of the HRC and adding the missing 1151 source identifications.

\section{Classification criteria and object categories}

A detailed description of the classification criteria has been given in Paper I. Here we shortly summarize the classification criteria of the various spectral classes. Sample spectra are shown in the Appendix.

Spectral classification was performed on the basis of the appearance of both the high-resolution density spectra and the images on the direct plates. The majority of optical objects in the RASS error circles were point sources. Objects with blue continua and occasionally emission lines dominated. Most of them are Seyfert galaxies or QSO. A minority of blue objects was classified as cataclysmic variable. They show the typical Balmer and $\mathrm{He}$ emission lines whereas AGN exhibit only few and usually broad emission lines. A few blue objects showed the spectral features of white dwarfs. Other stellar sources were classified according to the spectral features listed in Table 1. Spectra of stars brighter than $\sim 12^{\mathrm{m}}-13^{\mathrm{m}}$ are saturated. Hence a spectral type could not be determined and they were classified as "SATURATE".

The classification as a galaxy required an extended direct image of the optical source and the spectral features of galaxies, in particular the presence of the Ca break. A subclass of galaxies showed a conspicuous blue continuum, leading to the classification as "BLUE GALAXY".

Optically faint objects could not be classified into specific classes due to the low $\mathrm{S} / \mathrm{N}$ ratio of the spectra. For these spectra three categories were used corresponding to the continuum properties. The class "EBL-WK" is characterized by weak but extremely blue spectra. The opposite are objects with weak red spectra. They were classified "RED-WK". Intermediate cases were classified as "BLUE-WK". Most EBL-WK and BLUEWK are Sy 1 galaxies or QSOs. This was verified by slit spectra obtained during follow-up observations, e.g. Engels et al. (1998).

Likewise, BL Lac objects could not individually be classified as such with our prism spectra. They are expected to appear mostly in the class of AGN, QSO, EBL-WK or BLUE-WK due to their usually blue continua. However, a small fraction probably occurs in the class of RED-WK as in few cases BL Lac spectra were found to be rather red (e.g. Perlman et al. 1996).

In the second step the spectral classifications were used to assign an object category to the counterpart of the X-ray source. These categories are designated by one of the identification codes listed in Table 2 . For each identification a reliability flag was added which can take the values 0,1 or 2 for "highly probable", "probable", and "possible" identifications, respectively (cf. Paper I). The identification process included the valuation of the distance between X-ray and optical source, X-ray hardness ratios, X-ray source extension, and X-ray to optical flux ratios.

X-ray sources with counterparts of classes EBL-WK or BLUE-WK usually were combined with Sy galaxies and QSOs 
Table 1. Object classes used for the classification of the prism spectra of objects found in the X-ray error circles.

\begin{tabular}{ll}
\hline \hline Class & Description \\
\hline W-DWARF & white dwarf, blue continuum, broad absorption lines \\
CV & cataclysmic variable, exhibits Balmer emission \\
STAR-BA & Balmer line absorption \\
STAR-FG & G-band, Ca H\&K absorption \\
STAR-K & G-band, Ca H\&K absorption, redder continuum than STAR-FG \\
STAR-M & continuum very red, TiO and CaI 4226 A absorption \\
GALAXY & red continuum, no emissison lines, extended direct image \\
BLUE GALAXY & continuum blue, extended direct image \\
AGN & continuum blue, emission lines \\
QSO & continuum very blue, point-like image, emission lines \\
EBL-WK & continuum very blue, faint point-like image \\
BLUE-WK & blue continuum, faint point-like image \\
RED-WK & red continuum, faint point-like image \\
UNIDENT & none of the above defined classes assignable \\
OVERLAP & no classification possible due to overlapping spectra \\
SATURATE & no classification possible due to saturated spectrum \\
\hline
\end{tabular}

in a category designated "AGN" with ID code 1 . It comprises Sy, QSOs, and BL Lacs. Galaxies and blue galaxies are summarized in category "galaxy" with ID code 2 . The identification of a galaxy cluster (ID code 3) was based on the presence of several galaxies in the surroundings of the X-ray source position (cf. Paper I). X-ray properties like hard spectra or source extent often helped to improve the plausibility of the identification of an X-source with a galaxy cluster. For optically faint clusters there is some chance for misclassification as individual galaxy if a bright $\mathrm{cD}$ galaxy is present but most other members are below the plate limit of the blue direct plates (see also Sect. 5.1). Stars were identified according to their spectral class except the class "SATURATE" which were identified as "bright star" with ID code $7 \underline{x} 4$. Sources with no (unambiguous) identification or only RED-WK objects in the error circle were considered unidentified with ID code 8 . X-ray sources with a unique optical counterpart on the direct plates within $\approx 30^{\prime \prime}$ of the X-ray position, but fainter as the spectral plate limit, were separated using an ID code 803. In a considerable number of cases no object was visible on our blue plates within $40^{\prime \prime}$ around the RASS positions. These sources were designated "blank fields" and identified with ID code 0 .

\section{Description of the catalogue}

All information was collected in a catalogue containing X-ray and optical data together with the source identification. In Table 3 a section from the catalogue is displayed as an example. Each entry consists of at least 3 lines with X-ray data and data of the optical sources in the error circle. The optical source identified as the counterpart of the RASS source is flagged by a "+" sign preceding the running number of the optical source. In the case of blank fields the entry for optical sources is absent and therefore only two lines are displayed. The contents of the individual lines and columns is as follows:

1. The first line of each entry contains the X-ray data from the BSC (cf. Voges et al. 1999):

(a) 1RXS designation;
Table 2. Codes and object categories used in the HRC for the identification of X-ray sources. The identification reliability flag $\underline{x}$ can take the values $0=$ "highly probable", 1 = "probable", 2 = "possible".

\begin{tabular}{|c|c|}
\hline ID code & Object category \\
\hline $1 \underline{x}$ & $\begin{array}{l}\text { active galactic nucleus (“AGN") } \\
\text { including Sy, QSO and BL Lac }\end{array}$ \\
\hline $2 \underline{x}$ & galaxy \\
\hline $3 \underline{x}$ & galaxy cluster \\
\hline $5 \underline{x}$ & M star \\
\hline $6 \underline{\bar{x}}$ & white dwarf \\
\hline $7 \underline{-} 1$ & K star \\
\hline $7 \underline{x} 2$ & F-G star \\
\hline $7 \underline{-} 3$ & cataclysmic variable \\
\hline $7 \underline{x} 4$ & bright star \\
\hline $8-$ & no (unambiguous) identification, overlaps \\
\hline 803 & no spectrum available for likely counterpart \\
\hline 0 & $\begin{array}{l}\text { no objects visible within } 40 \text { " around the X-ray } \\
\text { position ("blank field") }\end{array}$ \\
\hline
\end{tabular}

(b) count rate and error in $\mathrm{cts} \mathrm{s}^{-1}$;

(c) hardness ratios with errors $H R 1, \sigma(H R 1), H R 2$, $\sigma(H R 2)$;

(d) source extension and extension likelihood.

2. Second line:

(a) RASS name as used in the first catalogue version;

(b) right ascension and declination (J2000.0) of the X-ray source;

(c) nominal $90 \%$ error circle as provided by the SASS processing;

(d) internal identifier of the HRC data base;

(e) identification code of the source according to Table 2;

(f) number of modifications of the identification;

(g) date of identification/last modification.

3. Third and following lines:

(a) running number of objects visible in the error circle; 
(b) right ascension and declination (J2000.0) of the optical source;

(c) positional offset in arcsec between X-ray and optical source in right ascension and declination;

(d) distance between X-ray and optical source in arcsec;

(e) $B$ magnitude;

(f) $B-R$ colour from USNO-A 2.0 catalogue (Monet et al. 1998);

(g) spectral class according to Table 1.

$B$ photometry was usually taken from the HQS. The HQS $B$ magnitudes are calibrated in the Johnson system (Engels et al. 1994). In cases of bright stars, however, the HQS photometry based on the low-resolution scans of the prism plates did not allow to measure the $B$ magnitude because of saturated spectra. We then searched the USNO catalogue for the corresponding entry and adopted the USNO $B$ magnitude if a matching entry was found within 5 arcsec. Due to proper motion effects this was not always the case. We then enlarged the search radius to 10 arcsec. In this case matching objects were flagged by the letter "p" following the $B$ magnitude. If no match was found even within 10 arcsec only a limit of " $<10.0$ " is given for the respective object magnitude.

Occasionally source confusion caused problems in the automatic assignment of $B$ magnitudes from the HQS for a faint object located close to brighter objects. In order to detect these cases we compared our $B$ magnitudes with that in the USNO catalogue and substituted USNO values if the HQS magnitude was brighter by at least $0.7 \mathrm{mag}$. These magnitudes are flagged by the letter " $u$ ". A correction of +0.4 was applied to the USNO magnitudes in order to transform the photographic $O$ magnitude of the USNO to Johnson $B$ (Mickaelian et al. 2001). The $B-R$ colours included in the HRC are all taken from the USNO catalogue.

\section{Discussion}

\subsection{Source statistics}

In Table 4 the statistics of the final identification of the 5341 RASS-BSC sources is summarized. For $82.2 \%$ an identification could be found. In $2.9 \%$ of the X-ray sources no optically visible objects were found within $40^{\prime \prime}$ around the X-ray source position (blank fields). Another 14.9\% remained unidentified, partly due to unusable spectra e.g. because of overlapping spectra, but mostly due to optically faint candidates with spectra of too low $\mathrm{S} / \mathrm{N}$ ratios.

Experience from follow-up spectroscopy and comparison with the literature shows that misidentifications are $<10 \%$. Therefore, on a case-by-case basis each of our identifications requires confirmation by optical slit spectroscopy, and is strictly speaking providing a candidate. This should be kept in mind in the following discussions, where we do not explicitly consider the statistical uncertainty of our identifications.

The largest group of X-ray emitters are the active galactic nuclei comprising $41.5 \%$ of all sources. Further extragalactic sources are $4.5 \%$ galaxies and $4.9 \%$ clusters of galaxies. The second largest group are the bright stars with a share of $22.8 \%$. F-G stars only contribute $0.8 \%$ due to the fact that most of

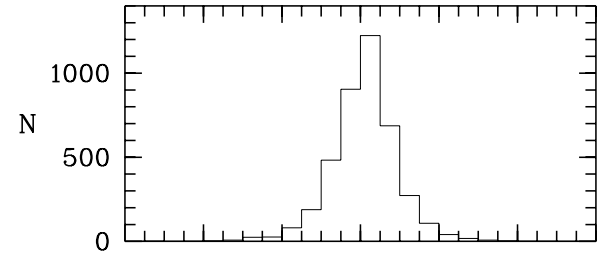

all sources
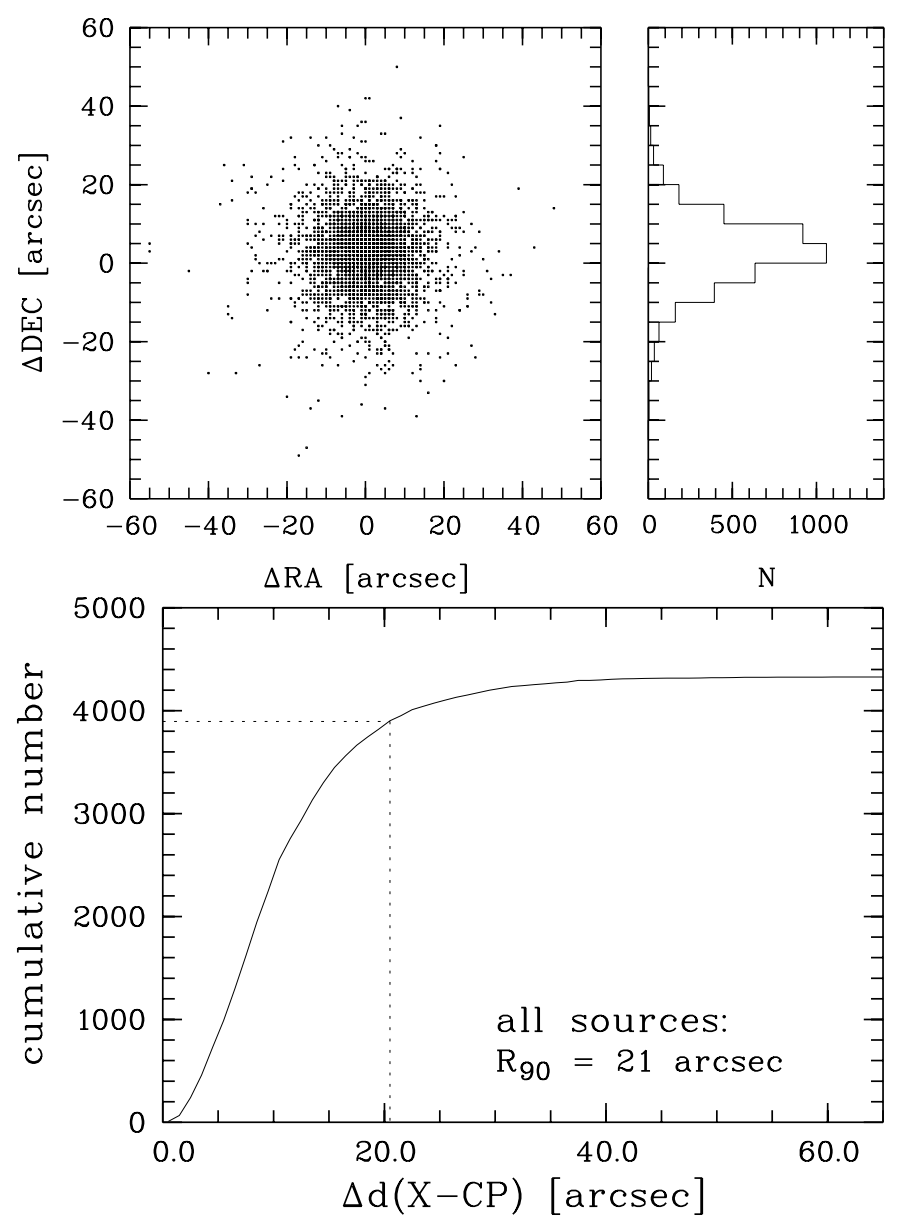

Fig. 1. Upper panel: difference between RA and Dec, respectively, of the X-ray and optical position for all point-like counterparts. The histograms show the frequency distribution of the positional offsets. No systematic effects are discernible. Lower panel: cumulative number of sources plotted over distance between optical counterpart and Xray position. $90 \%$ of the optical counterparts are within $21^{\prime \prime}$ from the $\mathrm{X}$-ray source.

these stars are so bright that their spectra are saturated and hence fall into the class of bright stars. $\mathrm{K}$ and $\mathrm{M}$ stars contribute $2.6 \%$ and $3.7 \%$, respectively. Likewise, small contributions of $0.8 \%$ and $0.5 \%$ are due to white dwarfs and cataclysmic variables, respectively. Hence we identified $\sim 51 \%$ with extragalactic sources and $\sim 30 \%$ with stellar coronal emitters.

These numbers can be compared with the results of other programs for optical identification of X-ray selected samples. We first consider two surveys based like our program on the RASS, i.e. on all-sky observations in the soft energy band. These are the LSW-INAOE-MPE survey (LSW survey hereafter) (Zickgraf et al. 1997; Krautter et al. 1999) comprising RASS sources down to count rates of $0.01 \mathrm{cts} \mathrm{s}^{-1}$ and 
Table 3. Section of the catalogue.

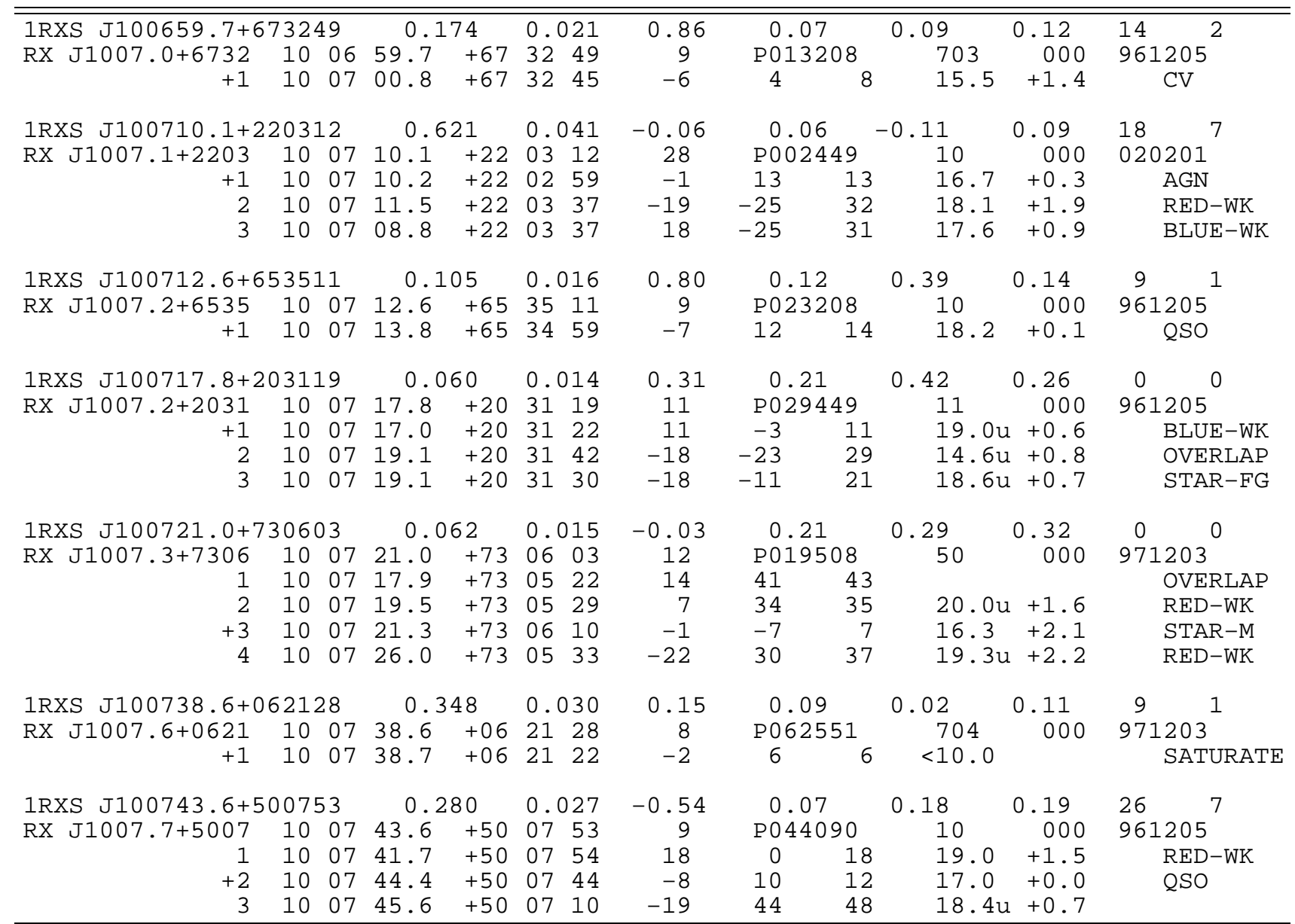

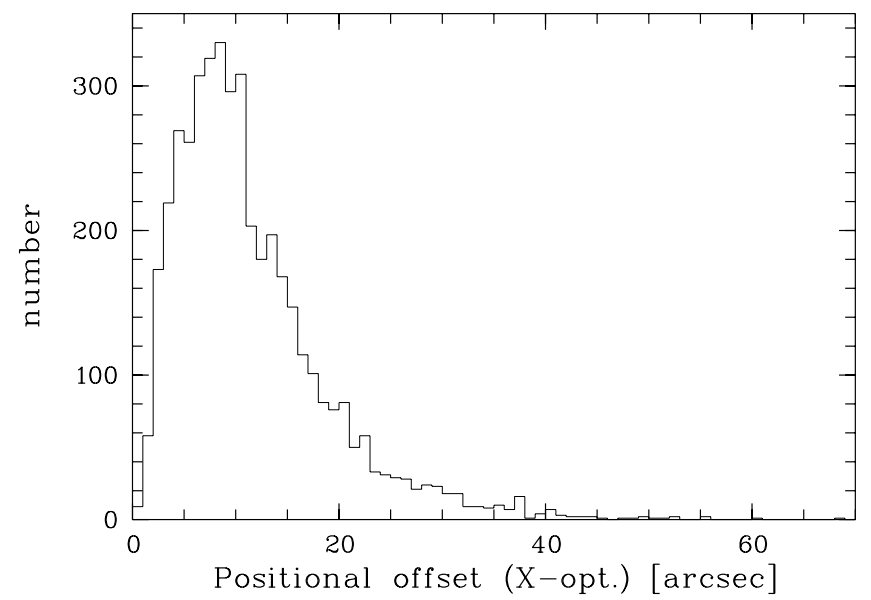

Fig. 2. Histogram of the positional offsets between X-ray position and position of the optical counterpart for all X-ray sources identified with optical point sources.

the NEP survey with an even deeper RASS exposure (Henry et al. 2001), reaching count rates as low as $1.2 \times 10^{-3} \mathrm{cts} \mathrm{s}^{-1}$ (Voges et al. 2001). Secondly, we compare our results with the EINSTEIN Medium Sensitivity Survey (EMSS) which in contrast to the RASS is based on serendipitous instead of allsky observations. Moreover, the energy band of the EMSS,
$0.3-3.5 \mathrm{keV}$, is harder than that of the RASS. Table 5 summarizes the comparison. In order to account for the contributions to the individual object classes in the HRC from unidentifed sources (ID codes 8 and 803) and blank fields (ID code 0) we use estimates derived below in Sects. 5.5.3 and 5.5.4 from the investigation of the hardness ratios of the different object classes. The fractions corrected with these estimates are given in Table 5 in an additional row for the HRC.

\subsubsection{Comparison with the LSW and NEP surveys}

For the comparison with the LSW survey we exclude area I of this sample. It has an unusually high column density of neutral hydrogen and a different source composition compared to the other five areas (cf. Zickgraf et al. 1997). This is probably due to contamination by the close Tau-Aur star-forming region located about $20^{\circ}$ north of area I.

The fraction of coronal emitters in our HRC sample appears smaller than in the reduced LSW sample, which contains $36 \%$ coronal sources compared to our $30 \%$. The difference is at least partly due to the difficulty of identifying the faint $M$ dwarfs on our blue prism plates. Many of these stars appear probably in the RED-WK class and are classified here as unidentified sources. Note, that $\mathrm{M}$ dwarfs in the BSC sample may be as faint as $B \sim 17.5$ to 18 , which is not far from the prism 
plate limit. Taking this into account by omitting the $\mathrm{M}$ dwarfs from the statistics leads to $26.2 \%$ coronal emitters in the HRC, and $25.8 \%$ in the LSW sample. Hence, no significant difference seems to be present between the HRC and the LSW survey for stellar sources with spectral types from $\mathrm{F}$ to K. For the NEP Henry et al. only give a total of $34 \%$ stars which contains an unknown fraction of $\mathrm{M}$ dwarfs. Correction for the estimated contribution of stars in the HRC from unidentified sources and blank fields yields a stellar fraction slightly larger than in the LSW and the NEP survey, namely $\sim 38 \%$ stars in the HRC versus $36 \%$ and $34 \%$, respectively. The indicated trend of a decreasing stellar fraction with decreasing flux limit could be due to the scale height of the stellar density distribution in the Milky Way. Note however that the differences are on a $2 \sigma$ level only.

Whereas we found $41.5 \% \mathrm{AGN}$, the reduced LSW sample has an AGN fraction of $46 \%$. The NEP survey with its even deeper RASS exposure yielded an even larger fraction of $51 \%$ AGN (including BL Lac objects) indicating an increasing AGN fraction with decreasing X-ray flux limit. Correction for the estimated contribution of AGN in the HRC from unidentified sources and blank fields, however, yields $\sim 49 \%$ of AGN. This value is between the AGN fractions in the LSW and NEP surveys of $46 \%$ and $51 \%$, respectively. Within the $2 \sigma$ limits of the LSW and the NEP survey no significant difference is found between the three surveys.

The galaxy fraction in the LSW survey, 4\%, was similar to our result. However, the fraction of galaxy clusters in the LSW sample is more than a factor of two larger (12\%). Actually, Krautter et al. (1999) found the highest fraction of galaxy clusters in the deepest exposed part of their RASS sample which is a factor of 5 more sensitive than the BSC sample. Furthermore, the optical CCD $R$ and $B$ images used by them to identify galaxy clusters reached fainter limiting magnitudes $\left(23^{\mathrm{m}}\right.$ and $22^{\mathrm{m}}$ in $R$ and $B$, respectively, cf. Zickgraf et al. 1997) than our blue plates which only reach as deep as $B \approx 20$. This explains the different fraction of galaxy clusters in the two samples. Likewise, the NEP survey contained $14 \%$ galaxy clusters, again significantly more than in the HRC, but only $0.2 \%$ galaxies. Like the LSW survey the NEP identification was also based on deep red CCD images. In addition the deep RASS-NEP exposure allowed to detect extended Xray sources alleviating the identification of galaxy clusters. A part of the missing clusters in the HRC could be hidden among the unidentified sources and the blank fields in particular. As will be discussed in Sects. 5.5.3 and 5.5.4 we expect in fact that a significant fraction of these sources in our BSC sample will be galaxy clusters. Taking the possible contributions from these sources to the galaxy cluster fraction into account (see Table 5) actually diminishes the differences with the LSW and NEP survey somewhat. With corrected $7 \%$ the differences relative to the LSW and NEP surveys are still $5 \%$ and $7 \%$, respectively. These differences are on a $4 \sigma$ level. Note, however that a fraction of the sources identified as galaxies could actually be galaxy clusters. This might e.g. be the case for clusters in which only the cD galaxy is visible on our direct plate. Adding the fractions of galaxies and galaxy clusters, no significant difference of the HRC (13\%), the LSW (16\%) and the NEP (15\%) is found. Taking these numbers and the possibility of misidentification into account we are led to the conclusion that the cluster fraction in the HRC is also not significantly different from the LSW and the NEP.

\subsubsection{Comparison with the EMSS}

Comparison with the EINSTEIN Medium Sensitivity Survey (EMSS) (Stocke et al. 1991) reveals small differences in the sample composition. The AGN fraction in the EMSS, 54\%, is significantly higher than in the uncorrected HRC with $42 \%$. For the corrected AGN fraction in the HRC, though still $~ 5 \%$ below the EMSS, the difference corresponds to only $\sim 2 \sigma$. On the other hand, stellar sources are less frequent in the EMSS (26\%) than in the HRC ( $31 \%$ uncorrected). The corrected stellar fraction of the HRC (38\%) is even $\sim 12 \%$ above the EMSS. This difference is significant on a $\sim 6 \sigma$ level.

With (uncorrected) $5 \%$ the fraction of galaxy clusters in the HRC would be significantly lower than the $12 \%$ in the EMSS. Note that in the EMSS as in the LSW and NEP surveys the galaxy cluster identification was based on deep red CCD images. Comparing with the corrected $7 \%$ the HRC still seems to be deficient in galaxy clusters relative to the EMSS. The difference is $\sim 4 \sigma$. As discussed above there is the possibility of galaxy clusters being identified as galaxies. Adding the fractions of galaxies and galaxy cluster together, no significant difference of the HRC (13\%) and the EMSS (15\%) is found. As for the LSW and the NEP we can thus state that the cluster fraction in the HRC is not significantly different from the EMSS.

Considering the number ratio of AGN and stellar coronae reveals a clear and significant difference between the EMSS and the RASS samples. The ratios (and their $1 \sigma$ uncertainties) are 2.08(0.03), 1.35(0.01), 1.29(0.01), 1.27(0.04), and 1.50(0.04) for EMSS, HRC, HRC $C$, LSW, and NEP, respectively. Hence the number ratio of AGN and stars in the EMSS is significantly different from the HRC and the other RASS based surveys.

A plausible explanation for this finding is the harder X-ray energy band of the EMSS compared to ROSAT. The stellar subsample consists mainly of coronal sources emitting predominantly in the soft X-ray band. They are thus expected to occur with a higher frequency in a survey at softer X-ray energies compared to harder energies. This has already been suggested by Gioia et al. (1990).

For the galaxy clusters the harder energy band of the EMSS seems not to play a dominant role for the detection efficiency although the energy spectra of galaxy clusters are hard. This follows from the similarity of the cluster fractions in the X-ray hard EMSS and the X-ray soft LSW and NEP surveys (see Table 5).

Apart from effects due to the the energy bands the different AGN to star ratios could also at least partly be caused by the selection of the X-ray sources from the IPC pointing of the EINSTEIN observatory. The EMSS is not an all-sky survey but consists of serendipitous X-ray sources in the neighborhood of X-ray bright objects. One fourth of the IPC fields used for the EMSS were pointing towards galactic objects. One third had an AGN as target. The targets of the pointings were excluded 
from the EMSS sample. This could introduce a bias in the sample composition. For a detailed discussion of the selection criteria of the EMSS sample and possible biases caused by the selection cf. Gioia et al. (1990).

Summing up, it may be said that the HRC agrees within the errors of the source statistics with the three other surveys except for the stellar fraction in the EMSS. This difference could be due to the harder EMSS energy band, the serendipitous character of this survey, or a combination of both.

\subsection{Positional accuracy}

In Fig. 1 the positional offsets of the optical counterpart and the X-ray position are plotted for right ascension and declination. No significant systematic shift is discernible in either coordinate. The lower panel of the figure depicts the cumulative distribution of distances between optical and X-ray position. We found that $90 \%$ of the point-like counterparts are found within $21^{\prime \prime}$ from the RASS position, i.e. the $90 \%$ error radius $r_{90}=21^{\prime \prime}$. This is significantly smaller than the $90 \%$ error radius determined from the LSW survey for which Krautter et al. (1999) give 30". The difference can be understood in terms of the better photon statistics for the BSC sources compared to the low count numbers of the RASS sources in the LSW sample. The frequency distribution of distances is displayed in Fig. 2 showing that the most frequent distance is $\sim 9^{\prime \prime}$ with a broad wing towards larger separations.

\subsection{Optical brightness distribution}

The distribution of the $B$ magnitudes of the counterparts is displayed in Fig. 3. We have excluded galaxies because of the unreliable photometry of the optically extended objects. The brightness distribution of the AGN class comprising Sy galaxies, QSOs and BL Lacs spans the range $B \sim 14-20$ and peaks around $B \sim 17$. The stellar sources, excluding CVs, have a broader distribution from $B \sim 4$ to $B \sim 18$ with a gradual increase and a maximum appearing around $B \sim 12-13$. The subgroup of white dwarfs (black histogram in Fig. 3) peaks around $B \sim 15$. CVs start around $B \sim 13$ and are found up to $B \sim 19$ with a peak at $B \sim 16$.

In Fig. 4 the brightness distribution for coronal emitters is broken down into the different contributions by F-G, K, M, and bright stars. Beyond the pronounced maximum the number of stars decreases in a broad wing towards fainter magnitudes.

\subsection{X-ray-to-optical flux ratio}

In order to determine the ratio of X-ray to optical flux for the various object classes we calculated the flux in the $0.1-2.4 \mathrm{keV}$ energy range from the BSC count rates. Conversion factors were determined with the WebPimms tool (version v3.2d) provided by HEASARC (Mukai 1993). For extragalactic sources column densities of neutral hydrogen, $N_{\mathrm{H}}$, were obtained from Dickey \& Lockman (1990) using EXSAS (Zimmermann et al. 1994). The column density distribution is displayed in Fig. 5. It peaks around $1.5 \times 10^{20} \mathrm{~cm}^{-2}$ with a wing up to $\sim 1.0 \times$
Table 4. Statistics of the identifications. "code" is the identification code used in the catalogue. The corresponding object class is listed in column "class". The absolute number and fraction in percent are given in the last two columns.

\begin{tabular}{llll}
\hline \hline Code & Class & Number & Fraction [\%] \\
\hline 0 & blank field & 155 & 2.9 \\
1 & AGN & 2215 & 41.5 \\
2 & galaxy & 238 & 4.5 \\
3 & galaxy cluster & 262 & 4.9 \\
5 & M star & 197 & 3.7 \\
6 & white dwarf & 45 & 0.8 \\
$7 \_1$ & K star & 141 & 2.6 \\
$7 \_2$ & F-G star & 45 & 0.8 \\
$7 \_3$ & CV & 26 & 0.5 \\
$7 \_4$ & bright stars & 1219 & 22.8 \\
8 & unidentified & 604 & 11.3 \\
803 & no spectrum & 194 & 3.6 \\
\hline Total: & & 5341 & 100 \\
\hline
\end{tabular}

Table 5. Comparison of the HRC statistics with the LSW-INAOEMPE ("LSW"), the NEP survey ("NEP"), and the EMSS. For each object class the percentage is given. Remarks: ${ }^{1}$ including BL Lac objects, ${ }^{2}$ without area I (Zickgraf et al. 1997), ${ }^{3}$ includes 3\% blank fields. In row "HRC $\mathrm{C}_{\mathrm{C}}$ " the unidentified sources and blank fields have been redistributed into the classes AGN, galaxy cluster, galaxies, and stars based on hardness ratio distributions. $1 \sigma$ errors are given in parentheses.

\begin{tabular}{llllll}
\hline \hline Survey & AGN $^{1}$ & Gal. & Gal. cl. & Stars & Unid. \\
\hline HRC & $42(0.9)$ & $5(0.3)$ & $5(0.3)$ & $31(0.8)$ & $18^{3}(0.6)$ \\
HRC $_{C}$ & $49(1.0)$ & $6(0.3)$ & $7(0.4)$ & $38(0.8)$ & - \\
LSW $^{2}$ & $46(2.6)$ & $4(0.8)$ & $12(1.3)$ & $36(2.3)$ & $2(0.5)$ \\
NEP & $51(3.4)$ & $1(0.5)$ & $14(1.8)$ & $34(2.8)$ & $1(0.5)$ \\
EMSS & $54(2.5)$ & $3(0.6)$ & $12(1.2)$ & $26(1.8)$ & $4(0.7)$ \\
\hline
\end{tabular}

$10^{21} \mathrm{~cm}^{-2}$. The median of the distribution is $2 \times 10^{20} \mathrm{~cm}^{-2}$ and the mean $<N_{\mathrm{H}}>=2.6 \times 10^{20} \mathrm{~cm}^{-2}$. For AGN we used a power law photon index of $\Gamma=2.5$ (see below). For individual galaxies and clusters of galaxies thermal bremsstrahlung with a temperature of $T=1 \mathrm{keV}$ and $T=8 \mathrm{keV}$, respectively, was adopted. For stellar sources we adopted $N_{\mathrm{H}}=10^{18} \mathrm{~cm}^{-2}$. Except for white dwarfs a Raymond-Smith model with $T=$ $1 \mathrm{keV}$ was used. The conversion factor for white dwarfs was determined for a black body energy distribution with $T=$ $50000 \mathrm{~K}$.

The optical fluxes in the $B$ band were obtained with the flux calibration for Johnson $B$ of $F_{B}\left(0.0^{\mathrm{m}}\right)=7.2 \times 10^{-9} \mathrm{erg} \mathrm{s}^{-1} \mathrm{~cm}^{-2}$ and a filter width of $\Delta B=1000 \AA$ (Lamla 1982). This results in the relation $\log f_{\mathrm{x}} / f_{B}=\log f_{\mathrm{x}}+B / 2.5+5.14$. The conversion to the usually used ratio $\log f_{\mathrm{X}} / f_{V}$ is given by $\log f_{\mathrm{x}} / f_{B}-\log f_{\mathrm{x}} / f_{V}=(B-V) / 2.5-0.36$ with $F_{V}\left(0.0^{\mathrm{m}}\right)=$ $3.92 \times 10^{-9} \mathrm{erg} \mathrm{s}^{-1} \mathrm{~cm}^{-2}$ and $\Delta V=800 \AA$. Hence differences are small, -0.36 for $B-V=0.0$ and +0.24 for $B-V=+1.5$. 


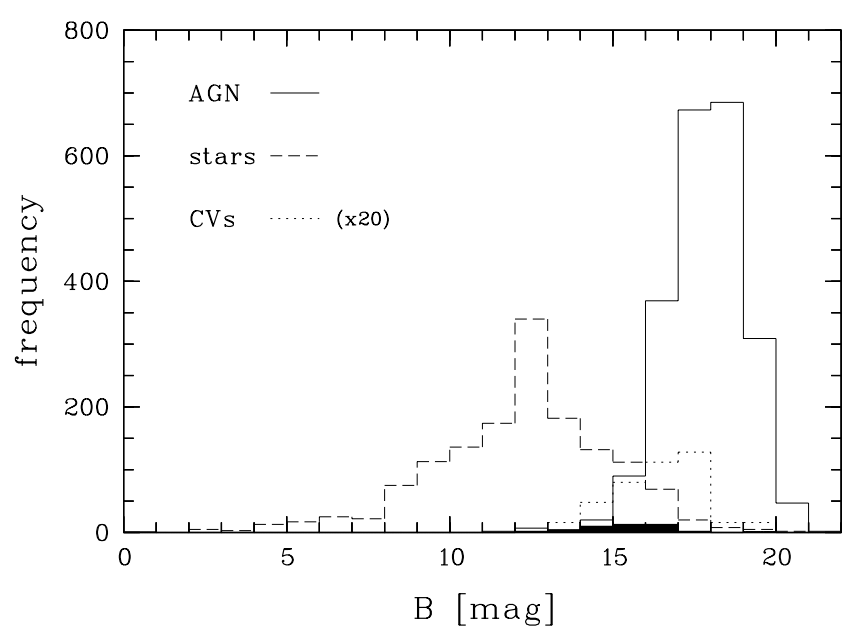

Fig. 3. Histograms showing the distribution of $B$ magnitudes for AGN, stars (bright stars, F-G, K, M stars, white dwarfs), and CVs. For the latter the ordinate scale has been expanded by a factor of 20 to account for the smaller numbers. The contribution of white dwarfs is additionally plotted as black filled histogram.

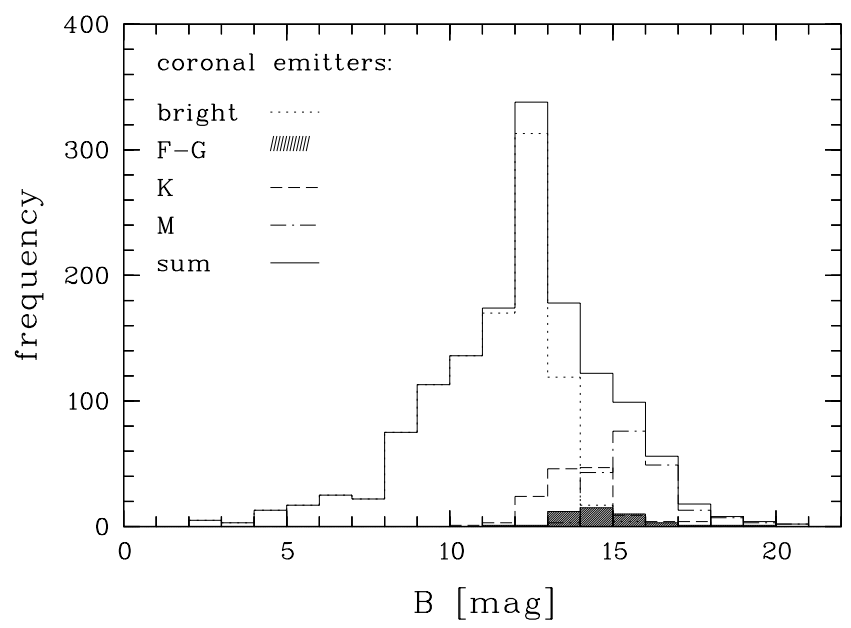

Fig. 4. Histograms showing the distribution of $B$ magnitudes for coronal emitters i.e. bright stars, F-G, K, and M stars. The small contribution from $\mathrm{F}-\mathrm{G}$ stars is shown as shaded histogram.

The resulting flux ratios $\log f_{\mathrm{x}} / f_{B}$ are listed in Table 6 and displayed in Fig. 6. For each object class the median and the limits of the frequency distributions at the $5 \%$ level, i.e. the range containing $90 \%$ of the sources, are given. For unidentified sources only in a few cases $B$ magnitudes of spectroscopically unidentified but likely optical counterparts could be listed in the catalogue with some confidence. Magnitudes of galaxies are uncertain due to the extended images. Likewise, only for a few galaxy clusters approximate $B$ magnitudes for the brightest members in the error circle could be used to obtain flux ratios. The ranges for these classes are therefore uncertain and are plotted as dotted lines. The flux ratios for galaxies and galaxy clusters are less reliable due to the selection effects and uncertain photometry for galaxies.

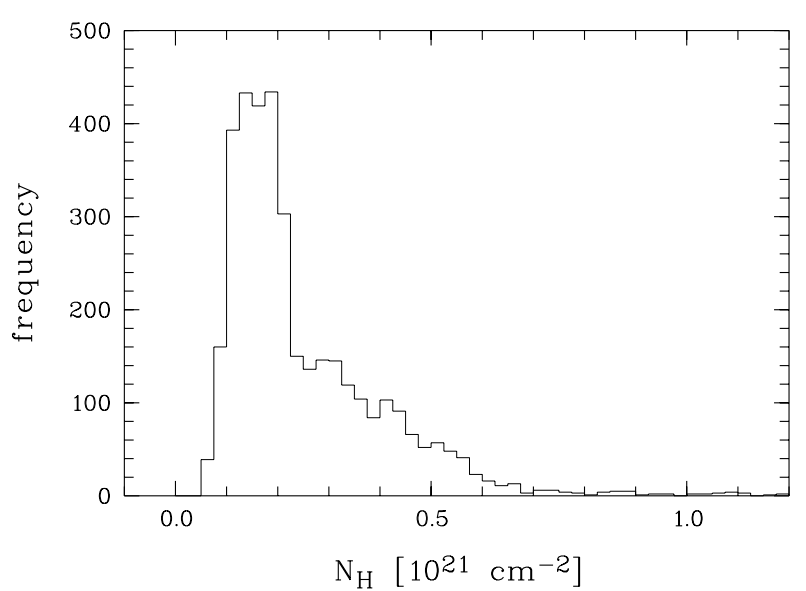

Fig. 5. Column density distribution of neutral hydrogen of extragalactic X-ray sources.

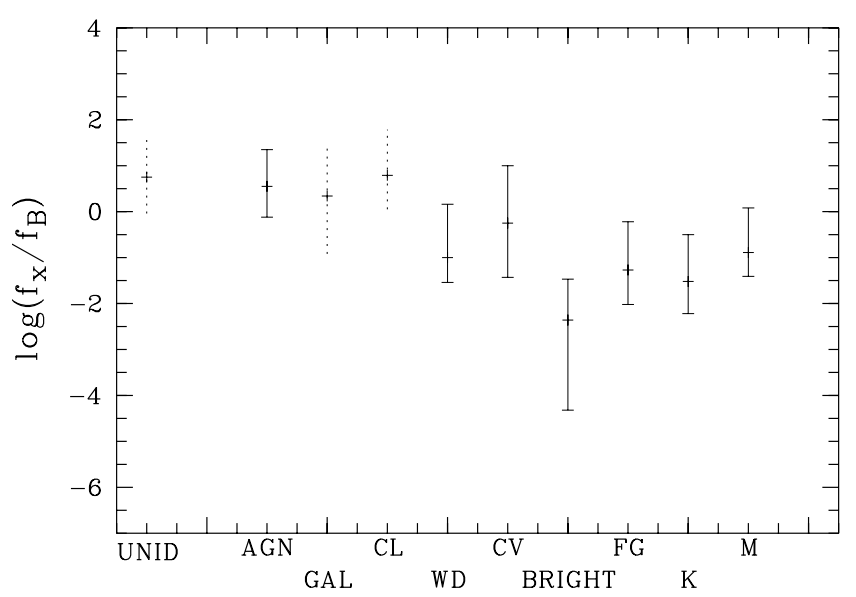

Fig. 6. X-ray-to-optical flux ratios for the different object classes. The diagram shows the range of $\log f_{\mathrm{x}} / f_{B}$ containing $90 \%$ of the counterparts and the median value of the respective class.

The median $\log f_{\mathrm{x}} / f_{B}$ for $\mathrm{AGN}$ is +0.55 . Accounting for the colour correction $(+0.16$ for $B-V=0.5)$ this is in very good agreement with the result of Krautter et al. (1999) of $<\log f_{\mathrm{x}} / f_{V}>=+0.44$ for the LSW sample. Among the stellar counterparts CVs have the highest flux ratio, $<\log f_{\mathrm{x}} / f_{B}>=-0.25$. Their flux ratio range overlaps partly that of AGN. Krautter et al. found a flux ratio of $<\log f_{\mathrm{x}} / f_{V}>=+0.42$ for the class of emission-line stars which contains mainly CVs. This is consistent with our range of flux ratios. White dwarfs are about on the same level as $M$ dwarfs which among the coronal emitters exhibit the highest flux ratio. Figure 6 suggests a trend of increasing $<\log f_{\mathrm{x}} / f_{B}>$ from bright stars, $\mathrm{F}-\mathrm{G}$ stars and $\mathrm{K}$ stars to $\mathrm{M}$ dwarfs. Note, however that the group of bright stars contains mainly $\mathrm{F}-\mathrm{G}$ stars and should therefore be combined with these. The combined flux ratios of the two groups thus span the range from -4.32 to -0.22 . 
Table 6. Flux ratios $\log f_{\mathrm{x}} / f_{B}$ for the various object classes. Column 2 lists the median value and Col. 3 the range containing $90 \%$ of the sources.

\begin{tabular}{llll}
\hline \hline \multirow{2}{*}{ Class } & $<\log f_{\mathrm{x}} / f_{B}>$ & \multicolumn{2}{c}{ Range } \\
& & Low & High \\
\hline AGN & +0.55 & -0.12 & +1.35 \\
galaxies & $+0.34:$ & $-0.92:$ & $+1.45:$ \\
galaxy clusters & $+0.79:$ & $+0.05:$ & $+1.78:$ \\
bright stars & -2.36 & -4.32 & -1.47 \\
F-G stars & -1.27 & -2.02 & -0.22 \\
K stars & -1.52 & -2.22 & -0.50 \\
M stars & -0.89 & -1.41 & +0.08 \\
white dwarfs & -1.00 & -1.54 & +0.16 \\
CVs & -0.25 & -1.43 & +1.00 \\
unidentified & $+0.75:$ & $-0.04:$ & $+1.64:$ \\
\hline
\end{tabular}

\subsection{Hardness ratios}

In Figs. 7-10 the hardness ratio $H R 1$ is plotted versus $H R 2$ for the different classes. Hardness ratios are defined as

$H R 1=\frac{[B]-[A]}{[B]+[A]}$

and

$H R 2=\frac{[D]-[C]}{[D]+[C]}$,

where $[A]$ to $[D]$ are the count rates in the respective energy bands $A=0.11-0.41 \mathrm{keV}, B=0.52-2.01 \mathrm{keV}, C=$ $0.52-0.90 \mathrm{keV}$, and $D=0.91-2.01 \mathrm{keV}$.

\subsubsection{Extragalactic sources}

For AGN the distribution of $H R 1$ is broad with a maximum around -0.4 whereas the distribution of $H R 2$ is narrower and peaks at $H R 2=0.0$. Galaxy clusters show significantly harder spectra with $H R 1$ having a maximum at +0.8 . Likewise $H R 2$ is harder than for AGN, although less conspicuous, with a peak at +0.2 . The hardness ratio distribution of galaxies resemble that of galaxy cluster when comparing the maximum of $H R 1$, although the distribution of the galaxies has a flatter shape at smaller $H R 1$. This indicates that possibly a fraction of the hard sources identified with individual galaxies in reality are galaxy clusters.

\subsubsection{Stellar sources}

Considering bright stars and $\mathrm{F}-\mathrm{G}$ stars as one class the bulk of these objects has hardness ratios around 0 for both, $H R 1$ and $H R 2 . \mathrm{K}$ and $\mathrm{M}$ stars appear to have slightly softer $H R 1$ with a maximum around -0.2 . Cataclysmic variables have a broad distribution of $H R 1$ with more hard than soft sources and a peak around $H R 1 \approx+0.2$ whereas $H R 2$ has a narrower peak at +0.4 . All white dwarfs but one exhibit very soft $H R 1$ of -1 . The object with $H R 1=+0.4(\mathrm{RX} \mathrm{J1523.1+3053)}$ though showing the optical spectrum of a white dwarf could be a misidentification because an optically faint background galaxy cluster cannot be excluded.

\subsubsection{Blank fields}

The distribution of $H R 1$ of the blank fields has a shallow maximum around +0.2 and possibly a second peak near +0.8 . Likely counterparts to blank fields are optically faint galaxy clusters, individual galaxies, AGN and CVs. Given the X-ray flux limit and the upper limits of the X-ray-to-optical flux ratios of the various classes of X-ray emitters counterparts of other object classes would be visible on our blue plates at a limiting magnitude of $B_{\text {lim }}=20$. The overall shape of the blank field histogram of $H R 1$ can be approximated by scaling and adding the contributions of the four classes. This is shown in Fig. 11. For galaxy clusters and galaxies the combined histogram was used. The decomposition performed in this way suggests that about $30 \%$ of the blank fields are galaxies or galaxy clusters, 45\% are AGN, and $25 \%$ are CVs. The high CV contribution seems necessary in order to explain the broad peak around $H R 1 \approx+0.2$ which cannot be reproduced with AGN, galaxies, and galaxy clusters alone. It would thus imply that our sample contains possibly more than twice as much CVs as listed in Table 4.

\subsubsection{Unidentified sources}

The distribution of $H R 1$ for unidentified sources is broad and asymmetric with a steep decrease between $H R 1 \approx-0.4$ and -0.8 , a maximum around $H R 1 \approx 0.0$ and with more hard $(H R 1 \geq 0.0)$ than soft sources $(H R 1<0.0)$. We decomposed the distribution in a similar way as the blank fields. Galaxies and galaxy clusters were combined as before. Stellar counterparts were all summed up in one histogram for "stars". The third class contributing to the unidentified sources are AGN. The result of the decomposition is depicted in Fig. 12. Stars and AGN seem to contribute equal shares of about $40 \%$ to the unidentified sources whereas only a fraction of $20 \%$ is probably due to optically faint galaxy clusters and galaxies.

Taking the galaxy cluster and galaxy contributions from both, blank fields and unidentified sources, into account would increase the total fraction of galaxy clusters from $4.9 \%$ to $\sim 7 \%$ and the galaxies from $4.5 \%$ to $\sim 6 \%$. For these estimates the combined numbers of galaxy clusters and galaxies were divided according to their relative fraction among the identified sources. Likewise, the AGN fraction would increase to $49 \%$ and the stellar fraction to $38 \%$ (cf. Table 5).

\subsection{Continuum properties of AGN}

\subsubsection{Radio emission}

A basic property of AGN is their radio emission which is believed to be related to the presence of more or less prominent radio jets emerging from the central engine. Based on the ratio of radio to optical flux they are separated in radio-loud and radio-quiet AGN. Recent studies of radio-loud X-ray selected 

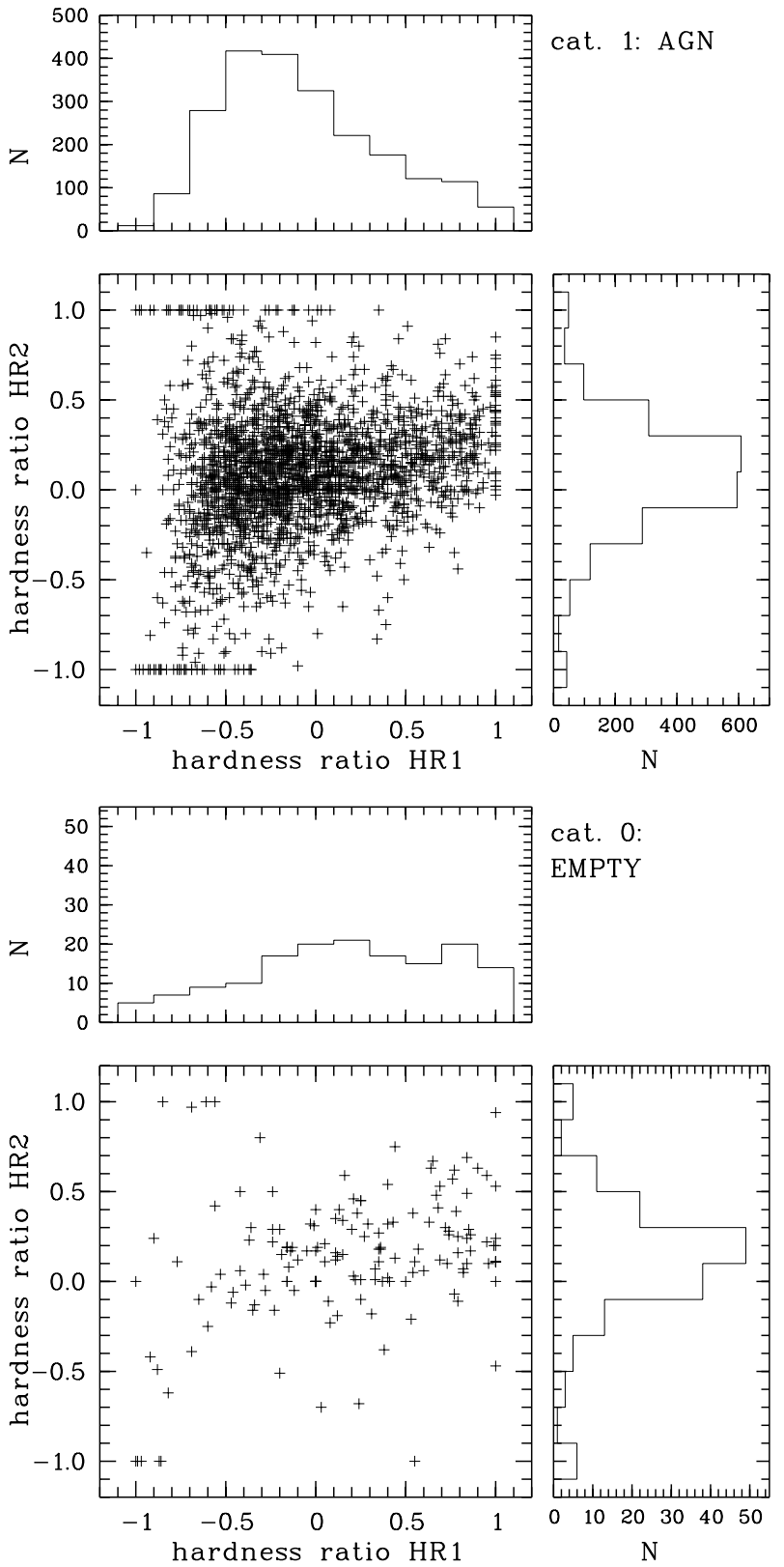

cat. 0 : EMPTY

cat. 1: AGN

EMPTY

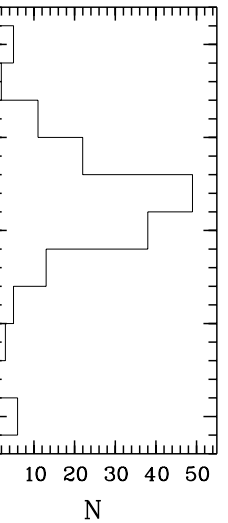

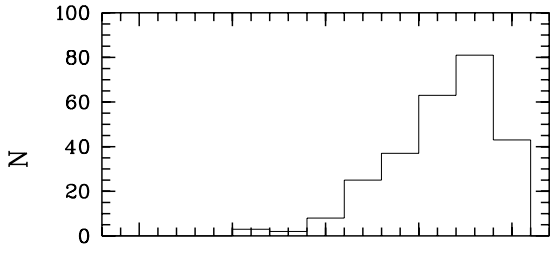

cat. 3:

GALAXY CLUSTER
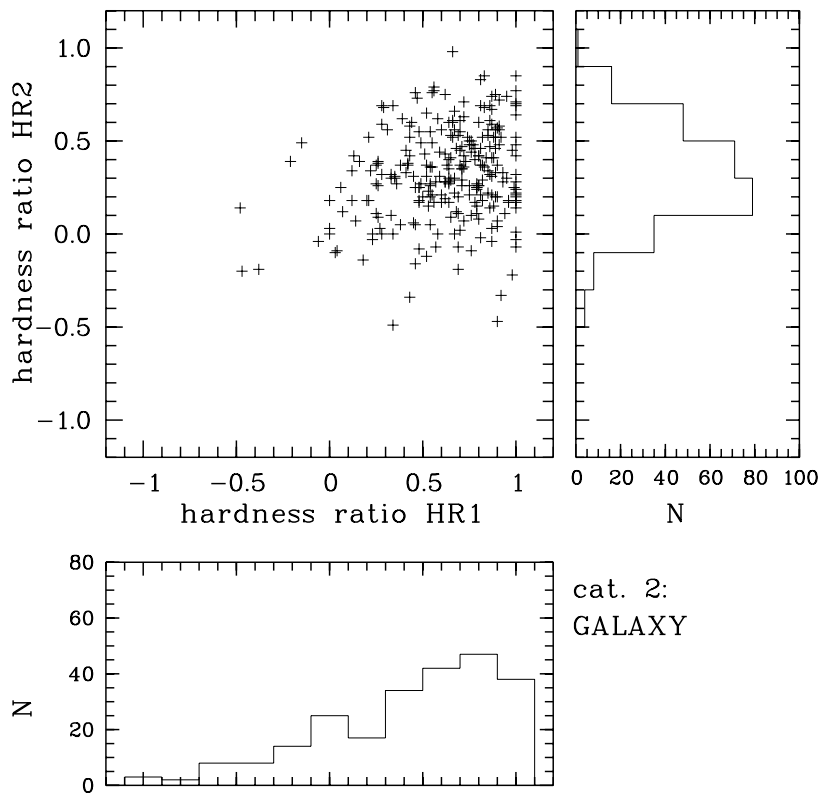

cat. 2:

GALAXY
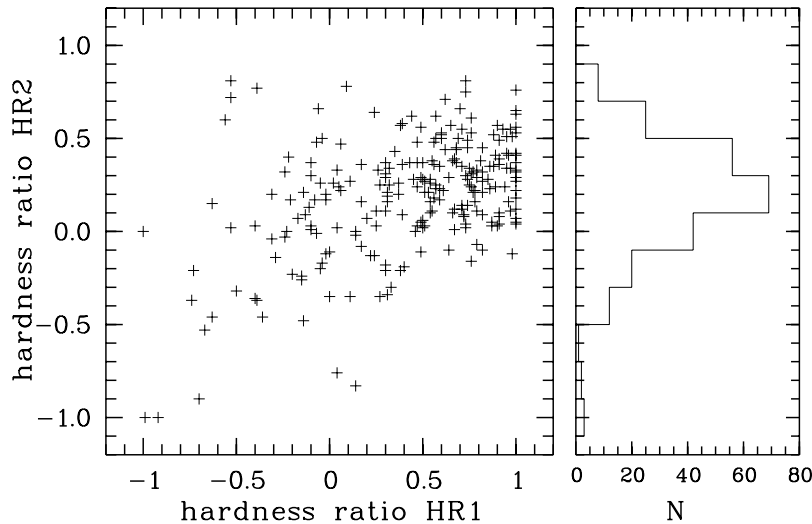

Fig. 7. Hardness ratios of extragalactic sources and blank fields ("EMPTY").

AGN have been carried out e.g. by Laurent-Muehleisen et al. (1997), Perlman et al. (1998), and Landt et al. (2001).

In their review on radio-loud AGN Urry \& Padovani (1995) quote a fraction of 15-20\% of radio-loud AGN. This fraction increases with optical and X-ray luminosity (Padovani 1993; La Franca et al. 1994; Della Ceca et al. 1994) to up to 50\%. Due to the lack of redshifts for most AGN from our HRC sample we cannot investigate the dependence of the radio-loud to radioquiet fractions on luminosity. We can, however, obtain at least a general picture of how both classes are represented in the HRC and how this compares with AGN samples selected by different methods.

In order to obtain radio fluxes for our AGN sample we searched in several steps the NRAO-VLA $1.4 \mathrm{GHz}$ (NVSS, Condon et al. 1998) and the Green Bank GB6 $4.85 \mathrm{GHz}$ (Gregory et al. 1996) catalogues for radio sources positionally matching the optical counterparts identified as AGN. The radio flux limits of these catalogues are $2 \mathrm{mJy}$ and $18 \mathrm{mJy}$, respectively. The search radii were initially set to $15^{\prime \prime}$ for NVSS and $80^{\prime \prime}$ for GB6. For the NVSS the number of matches was 559 of which $90 \%$ were found within $10^{\prime \prime}$. For the GB6 330 sources were detected with a 90\% radius of $58^{\prime \prime}$. Figure 13 depicts the histogram of the number of radio sources versus distance between NVSS radio and optical position (upper panel). In a second step we matched the NVSS and GB6 sources by searching coincidences within $40^{\prime \prime}$ of the NVSS sources. We found 183 positional matches. The histogram of distances is also shown in Fig. 13. Note, however, that the declination ranges covered by the two radio surveys are different. GB6 is limited to $0^{\circ} \leq \delta \leq+75^{\circ}$ whereas NVSS contains sources within $-40^{\circ} \leq \delta \leq+90^{\circ}$. For the coincidences we calculated the spectral index $\alpha_{\mathrm{r}}=1.85 \log \left(f_{4.85 \mathrm{GHz}} / f_{1.4 \mathrm{GHz}}\right)$, 

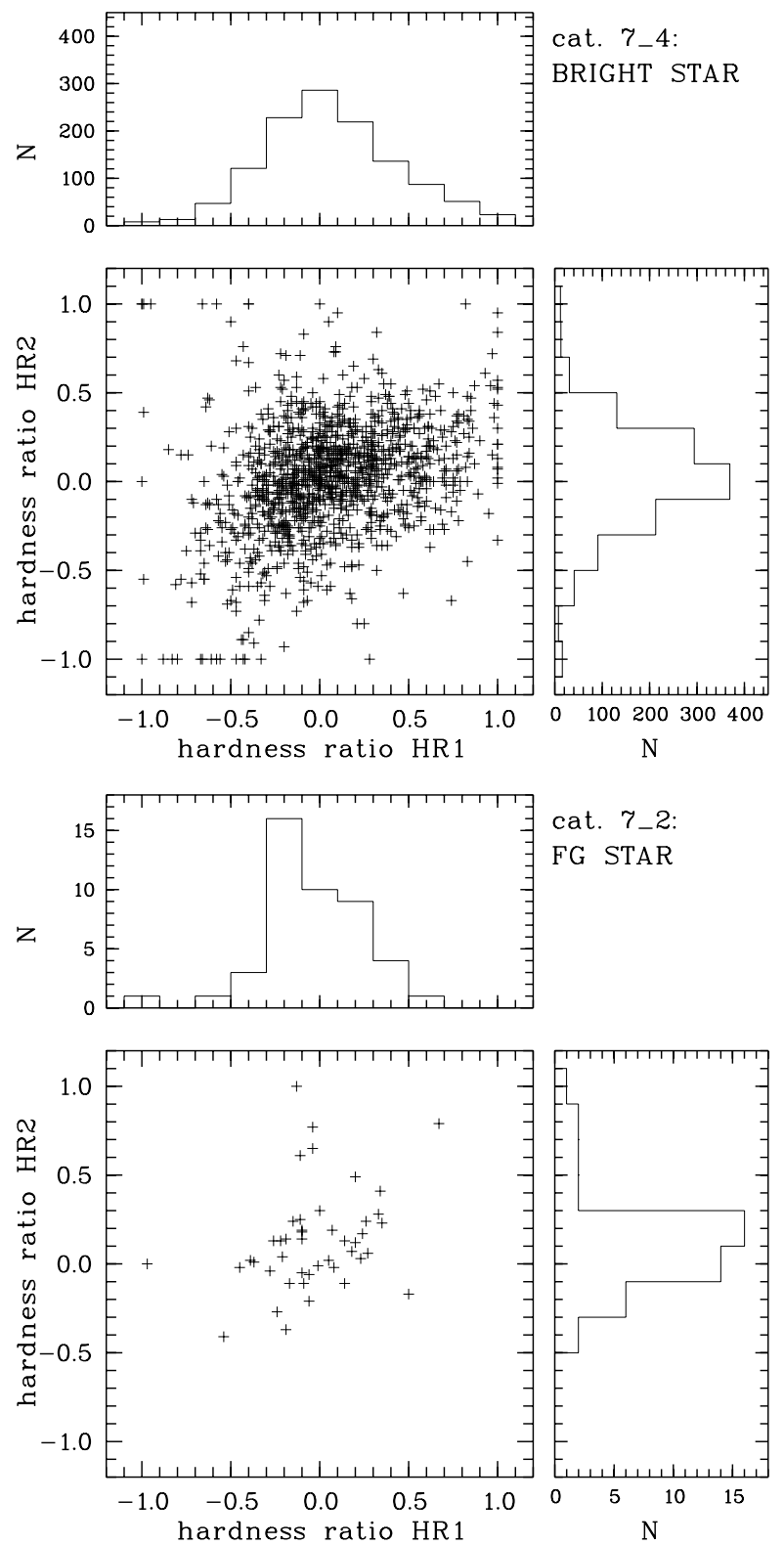

Fig. 8. Hardness ratios of bright stars, F-G, K, and M stars.

where $\alpha_{\mathrm{r}}$ is defined by $f_{v} \propto v^{\alpha_{\mathrm{r}}}$. The frequency distribution of $\alpha_{\mathrm{r}}$ has a maximum around +0.1 and covers a range from -1 to +1 .

The result of the the first step of the cross-correlation with the NVSS agrees well with that obtained by Bischoff \& Becker (1997) who searched for matches between the NVSS and known quasars. They give a $90 \%$ confidence error circle of $\sim 10^{\prime \prime}$ for a radio source at the flux limit of the survey and conclude that matches beyond $35^{\prime \prime}$ are almost totally chance coincidences. While this conclusion may be correct for core-dominated radio sources it may not hold for lobedominated sources. For these a larger search radius is appropriate. Our search for coincidences could thus miss lobedominated sources with core fluxes below the survey limit. In the DXRB survey Perlman et al. (1998) used a search radius of $90^{\prime \prime}$ in order to detect extended radio structure. Likewise, in the REX survey Caccianiga et al. (1999) used a large search
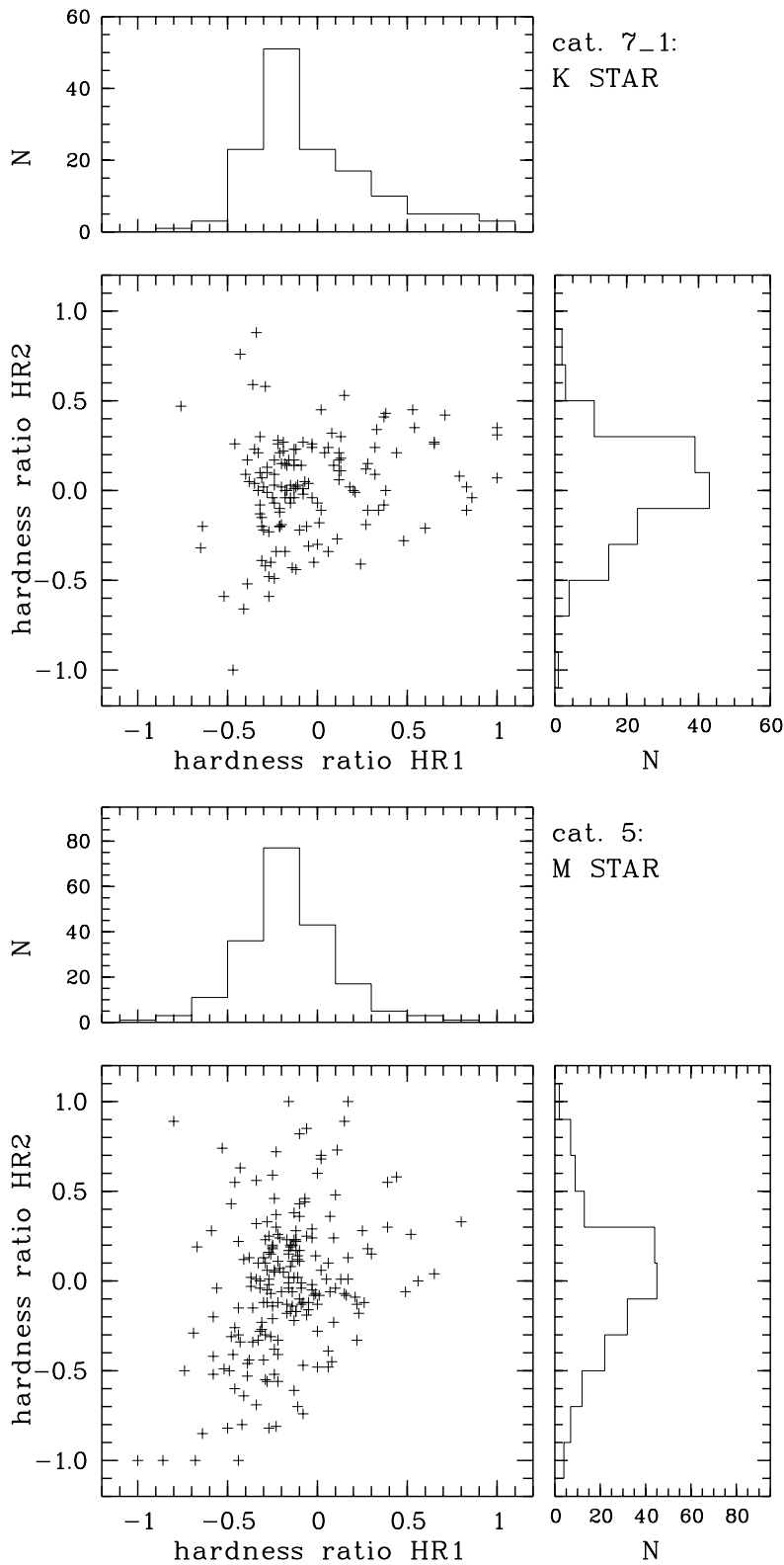

box for the same reason. In order to detect extended radio sources also in our sample we performed a further search step with a larger search radius of $90^{\prime \prime}$. For 812 RASS AGN candidates 1 to 3 NVSS sources were found within this radius. Of these 101 are double and 14 are triple radio detections. Among the double and triple sources 63 and 11, respectively, have one of the radio sources within $15^{\prime \prime}$ from the optical position. They were therefore already contained in the sample of 559 sources. The remaining 41 double and triple sources are new. The rest of 212 single sources has a separation larger than 15" between the optical and the radio source. Because their association with the RASS sources is questionable they were considered as chance coincidences.

The 41 new double and triple sources could be cases of lobe-dominated radio sources with a core flux below the survey limit. In order to test whether these radio sources are in fact related to the respective optical counterpart we checked 


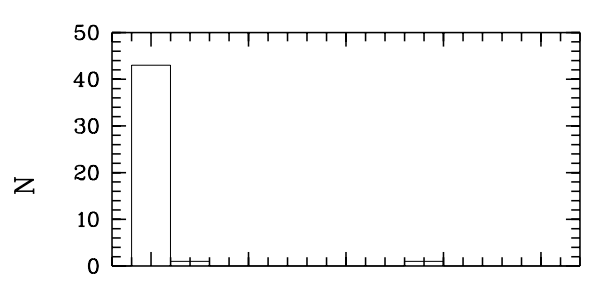

cat. 6:

WHITE DWARF
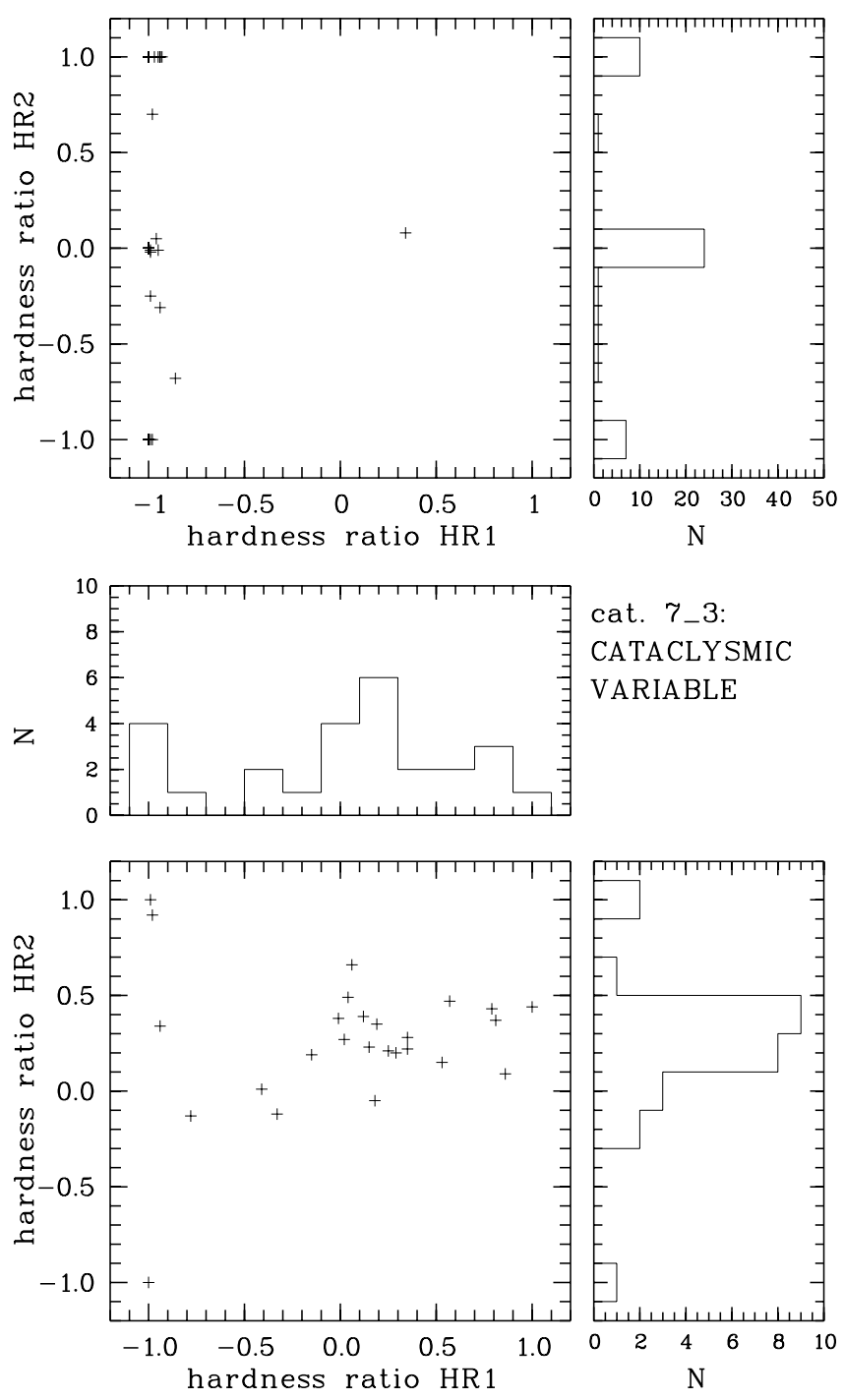

Fig. 9. Hardness ratios of white dwarfs and cataclysmic variables.

the angles between the optical and the radio positions (cf. also Caccianiga et al. 1999). Double lobe sources should have an angle difference of $180^{\circ}$. As test criterion we used the angle difference, allowing for an uncertainty of $\pm 25^{\circ}$. Certainly, a more detailed analysis would require to check the NVSS radio images individually. However, this is beyond the scope of this paper, and we restrict the discussion to the use of the angle difference. With this criterion the 11 triple sources with core emission qualify as double-lobe sources. Of the remaining 3 triple sources 2 are probably double-lobe sources without core emission. In these cases the third radio source seems to be unrelated to the optical object. One triple source is most likely not related to the optical counterpart of the X-ray source. For 11 double sources neither the angles difference nor the positional

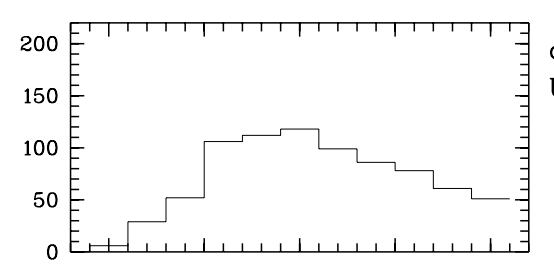

cat. 8:

UNIDENTIFIED

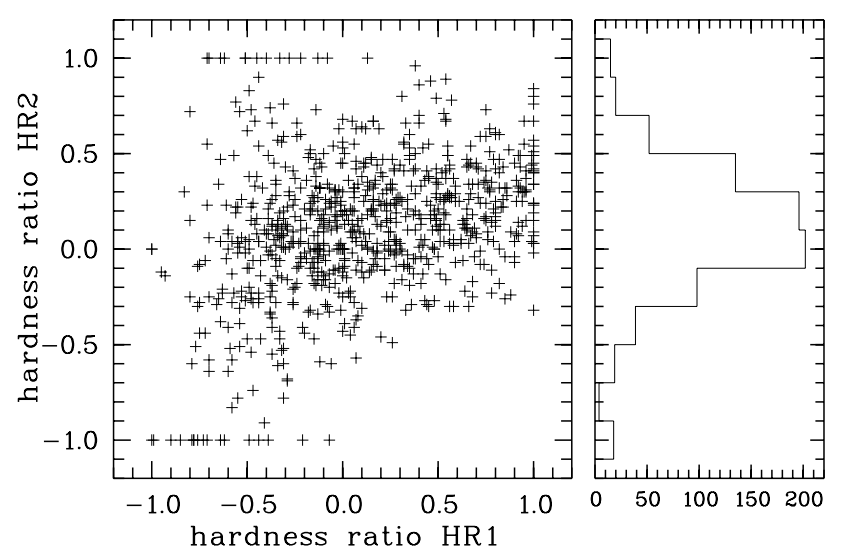

Fig. 10. Hardness ratios of unidentified sources.

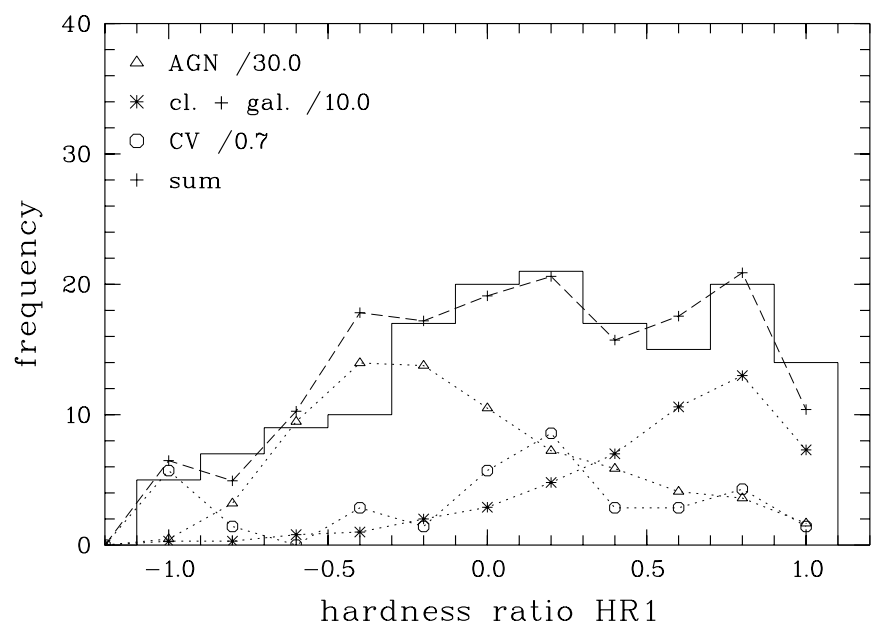

Fig. 11. Histogram of the hardness ratio $H R 1$ of blank fields (solid line). The dashed lines represent scaled frequency distributions of AGN, CVs, and galaxy clusters. The scaling factors given were chosen so that the sum of the scaled distributions (long dashed lines and + signs) approximates the observed histogram.

separation of the radio and the optical position indicates a relation between the radio sources and the optical object. For the remaining 27 cases the separations are between $168^{\circ}$ and $203^{\circ}$, in 22 cases even between $170^{\circ}$ and $190^{\circ}$. Based on the angle criterion the 28 sources can thus be classified as probable double-lobe sources without detected core emission. In summary, 29 of the 41 multiple sources can be assigned to the optical counterparts, and 12 are spurious detections. This means that the first search step with a $15^{\prime \prime}$ search radius missed $~ 5 \%$ of radio emitting AGN. In total, for 588 AGN radio emission was found. The results of the cross-correlation are summarized in Table 7.

In order to determine the radio-loudness we extrapolated the $1.4 \mathrm{GHz}$ fluxes to $8.4 \mathrm{GHz}$ and computed the ratio $R=f_{\mathrm{r}} / f_{\mathrm{o}}$ 


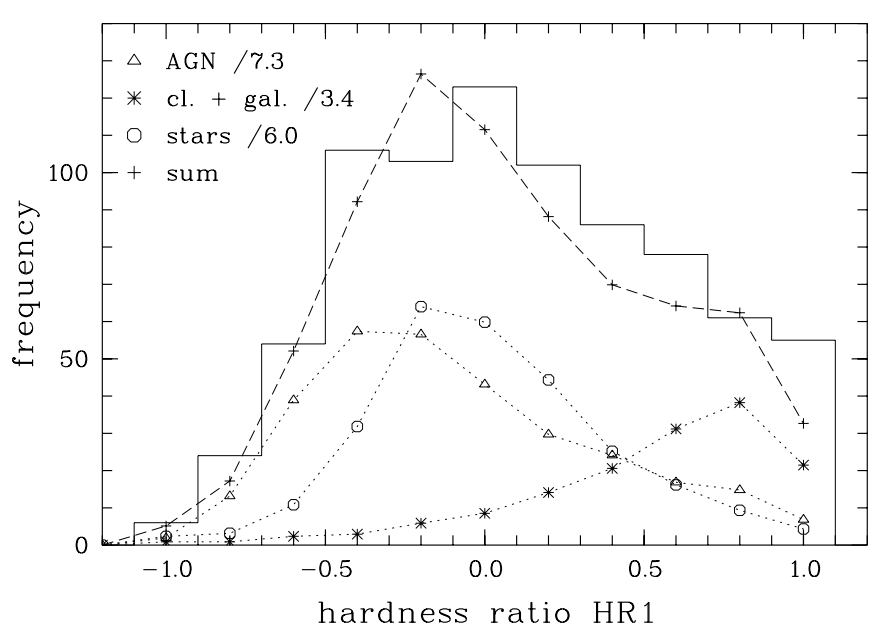

Fig. 12. Histogram of the hardness ratio $H R 1$ of unidentified sources (solid line). The dashed lines represent scaled frequency distributions of AGN, stars, and galaxy clusters. The scaling factors given were chosen so that the sum of the scaled distributions (long dashed lines and + signs) approximates the observed histogram.

with the $8.4 \mathrm{GHz}$ radio flux $f_{\mathrm{r}}$ and the monochromatic optical flux $f_{\mathrm{o}}$. The choice of $8.4 \mathrm{GHz}$ was made because some previous studies of the radio-loudness of quasars used this radio frequency to determine $R$, e.g. Hooper et al. (1996) for the Large Bright Quasar Survey (LBQS). The total radio flux of the multiple sources was determined by summing up the radio fluxes of the individual components. For sources without GB6 detection within $40^{\prime \prime}$ of the NVSS core source $\alpha_{\mathrm{r}}=-0.5$ was used for the extrapolation. Note that the results of the analysis of the radio-loudness do not depend significantly on the chosen frequency and hold as well for $1.4 \mathrm{GHz}$ and $4.85 \mathrm{GHz}$ radio fluxes. The optical flux, $f_{\mathrm{o}}$, for $\lambda=2500 \AA$ and magnitude $B$ was computed from the relation $\log f_{2500}=-22.55-0.4 B$ by Schmidt (1968). No K-correction was performed because the redshifts are unknown.

Radio-loud sources have $R>10$ (Kellermann et al. 1989). For AGN not detected in the NVSS nor found in GB6 an upper limit $R_{\text {lim }}$ was calculated by assuming an upper limit of $2 \mathrm{mJy}$ for the $1.4 \mathrm{GHz}$ radio flux. Radio-quiet AGN have either $R<$ 10 if radio flux is detected or $R_{\lim }<10$ otherwise. AGN not detected in the radio surveys are therefore only included in the radio-quiet sample if their $R_{\lim }<10$. For the remaining radio non-detections an assignment to eiter the radio-quiet or radioloud sample is not possible.

Of the 588 NVSS sources, 485 , i.e. about $22 \%$ of the entire AGN sample, have $R>10$ and hence are radio-loud. This fraction is a lower limit because taking into account the detection limit of $2 \mathrm{mJy}$ in the NVSS and the optical brightness of the faint AGN the sample of radio non-detections could contain further radio-loud sources. The frequency distribution of $R$ peaks around $R \approx 100$. All but four of the combined NVSS/GB6 matches are radio-loud. For these sources the mean radio spectral index is $\alpha_{\mathrm{r}}=+0.1$. Note that assuming a steep radio spectral index for a truly flat-spectrum source leads to an underestimated radio flux. Such a source could then fall into
$\mathrm{N}$
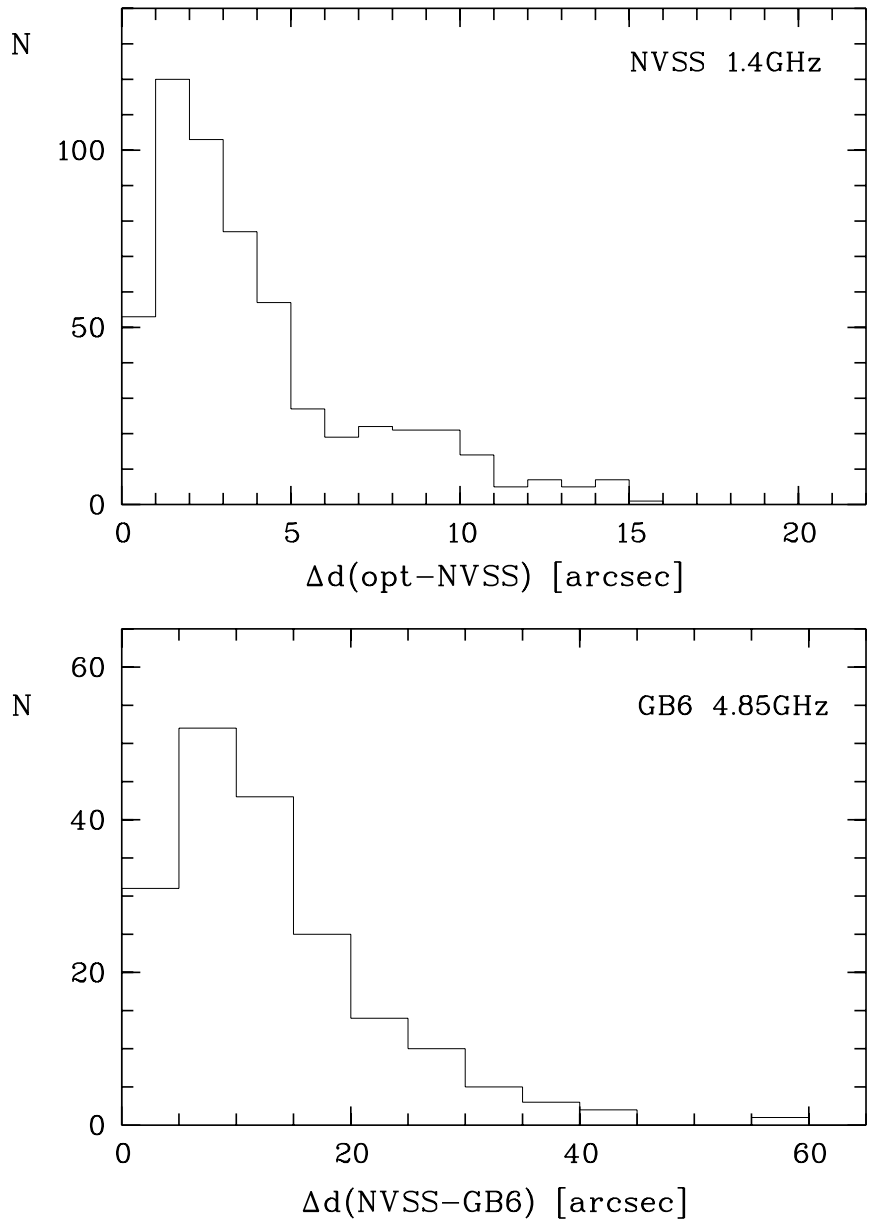

Fig. 13. Histograms showing the frequency of radio sources with given distances between optical counterpart and matching NVSS (upper panel) and between NVSS and GB6 radio sources (lower panel), respectively.

the group of radio-quiet AGN, hence leading to a decrease of the radio-loud fraction.

For the optically selected quasars from the LBQS Hooper et al. (1996) found a radio-loud fraction of $\sim 10 \%$. Hooper et al. furthermore give fractions of $11 \%$ and of $19 \%$ for quasars from the EMSS and from the Palomar-Green survey (PG) using radio data from Stocke et al. (1991) and Kellermann et al. (1989), respectively, extrapolated to $8.4 \mathrm{GHz}$. Our result of $\approx 22 \%$ radioloud AGN is similar to the PG sample, but twice as high as the LBQS and, in particular, the EMSS radio-loud fraction. The difference from the LBQS might be due to selection effects, as this optically selected sample contains mostly quasars and covers a much larger redshift range than the X-ray selected samples, which contain a much larger fraction of Seyfert galaxies. The reason for the difference from the EMSS is not as obvious since RASS and EMSS AGN have a comparable redshift distribution. However, one has to take into account that the EMSS is a serendipitous survey containing X-ray sources found in the vicinity of bright $\mathrm{X}$-ray sources. This might introduce a bias in the sample composition which could contribute to the differences in the radio-loud AGN fraction relative to an unbiased all-sky survey. 
Table 7. Results of the cross-correlation of the RASS AGN candidates and the NVSS. The type of NVSS source, distance between optical and radio position, $d$ (opt - radio), and the number $N$ of respective sources are given.

\begin{tabular}{llll}
\hline \hline Type of NVSS source & $d($ opt - radio $)$ & $N$ & Comment \\
\hline single & $d \leq 15^{\prime \prime}$ & 485 & core-dominated AGN \\
$(N=697)$ & $15^{\prime \prime}<d \leq 90^{\prime \prime}$ & 212 & association questionable \\
double & 1 component $d<15^{\prime \prime}$ & 63 & core-lobe AGN \\
$(N=101)$ & aligned & 27 & lobe-dominated AGN \\
& not aligned & 11 & association questionable \\
triple & 1 component $d<15^{\prime \prime}$ & 11 & core-lobe AGN \\
$(N=14)$ & aligned & 2 & lobe-dominated AGN \\
& not aligned & 1 & association questionable \\
\hline
\end{tabular}

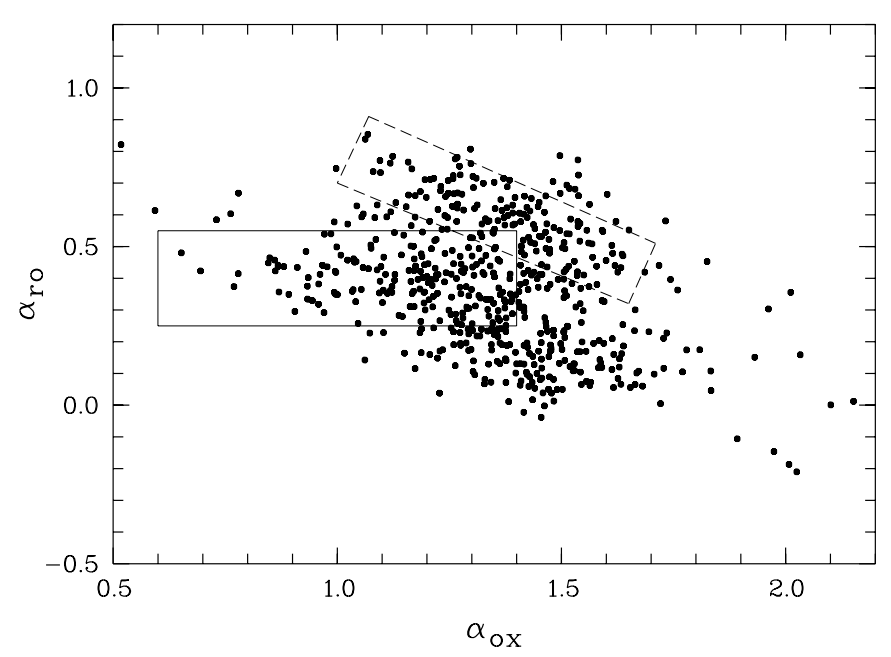

Fig. 14. Continuum slopes $\alpha_{\text {ox }}$ and $\alpha_{\text {ro }}$ for AGN. The index $\alpha_{\text {ro }}$ refers to a radio frequency of $4.85 \mathrm{GHz}$. The solid and dashed boxes showing the regions occupied by the two types of BL Lac objects, HBL and LBL, respectively, were adopted from Brinkmann et al. (1997).

Using 4.85 GHz radio fluxes, $f_{\mathrm{r}}$, either from GB6 or extrapolated from NVSS, monochromatic optical fluxes at $2500 \AA, f_{\mathrm{o}}$, and monochromatic X-ray fluxes at $2 \mathrm{keV}, f_{\mathrm{x}}$, for photon in$\operatorname{dex} \Gamma=2.5$ (see below) we calculated the continuum slopes $\alpha_{\text {ox }}=-\log \left(f_{\mathrm{x}} / f_{\mathrm{o}}\right) / 2.605$ and $\alpha_{\text {ro }}=\log \left(f_{\mathrm{r}} / f_{\mathrm{o}}\right) / 5.38$ as defined by Tananbaum et al. (1979) and flux ratios $f_{\mathrm{o}} / f_{\mathrm{r}}$ and $f_{\mathrm{x}} / f_{\mathrm{r}}$. Figure 14 shows the $\alpha_{\mathrm{ox}}-\alpha_{\text {ro }}$ diagram for the radiodetected AGN from the HRC.

A special class of radio-loud AGN are the BL Lac objects. Recent surveys aiming specifically at this class of objects were carried out e.g. by Laurent-Muehleisen et al. (1998, 1999), Rector et al. (2000), and Beckmann et al. (2003). Padovani \& Giommi (1995) suggested that BL Lacs can be separated into the subgroups of low-energy peaked (LBL) and high-energy peaked (HBL) objects. In the $\alpha_{\text {ox }}-\alpha_{\text {ro }}$ diagram we have indicated the regions in which these two types of BL Lacs are found. The parameter limits were taken from Brinkmann et al. (1997).

The flux ratios are depicted in Fig. 15 in a $\log \left(f_{\mathrm{x}} / f_{\mathrm{r}}\right)$ vs. $\log \left(f_{\mathrm{o}} / f_{\mathrm{r}}\right)$ diagram. As before the regions containing LBLs and HBLs are indicated. The limits for the flux ratio of $\log \left(f_{\mathrm{x}} / f_{\mathrm{r}}\right)$ are plotted as dashed lines in Fig. 15 and were adopted from

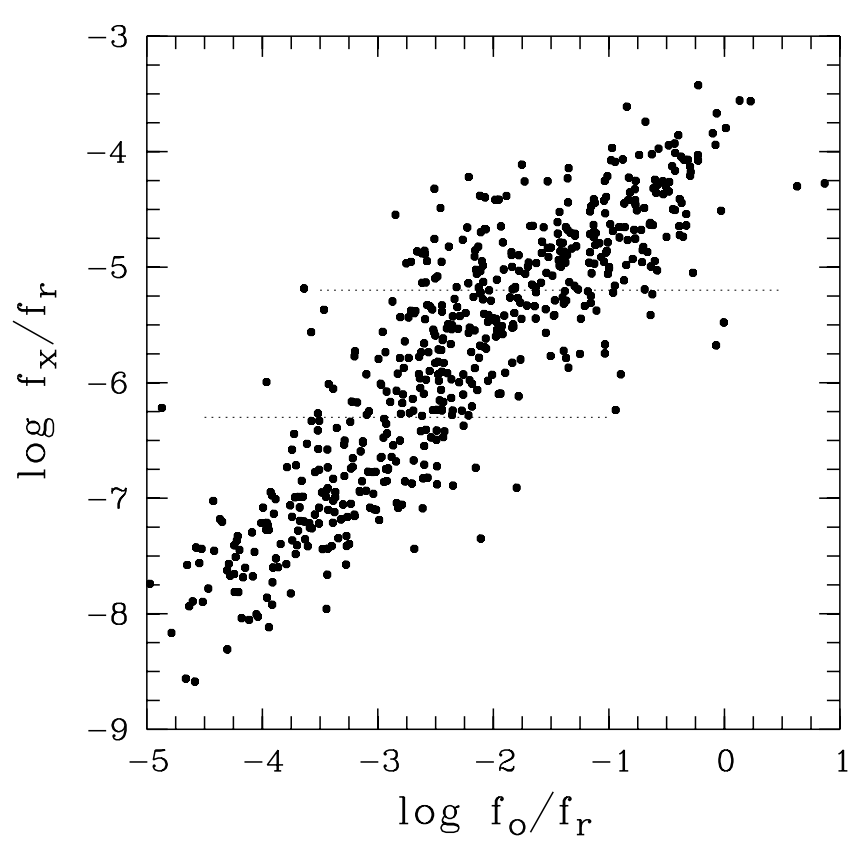

Fig. 15. Flux ratios for AGN. The dashed lines represent limits above and below of which HBL and LBL/HPQ, respectively, are found (limits adopted from Brinkmann et al. 1997).

Fig. 11 in Brinkmann et al. (1997). As discussed by Brinkmann et al. the class separation is more evident in the flux ratio diagram. The number of HBL candidates with $\log \left(f_{\mathrm{x}} / f_{\mathrm{r}}\right)>-5.2$ is 222. Below $\log \left(f_{\mathrm{x}} / f_{\mathrm{r}}\right)=-6.3186 \mathrm{LBL}$ candidates and candidates for highly polarized quasars (HPQ) are found. Which of the candidates are BL Lacs cannot be decided with our lowresolution prism spectra. The spectroscopic confirmation requires higher spectral resolution. This has partly been done in spectroscopic follow-up studies of BL Lac candidates from the HRC by Bade et al. (1998b) and Beckmann et al. (2003) who studied a sample of nearly 100 BL Lac objects. Although the total fraction of BL Lacs in the HRC therefore remains uncertain at this time we can at least conclude that obviously less than $10 \%$ of the AGN in our sample are HBL-type BL Lacs.

\subsubsection{X-ray photon index $\Gamma$}

The X-ray hardness ratios $H R 1$ and $H R 2$ can be used to obtain some information about the shape of the energy 
distributions in the $0.1-2.4 \mathrm{keV}$ range for AGN (and coronal emitters, see Sect. 5.7). For AGN we approximated the continuum shape $f(E)$ by a power law $f(E) \propto E^{-\Gamma}$ with the photon energy $E$ and the photon index $\Gamma$. Galactic foreground absorption due to neutral hydrogen was taken into account according to Morrison \& McCammon (1983) with $N_{\mathrm{H}}$ fixed at the value obtained from Dickey \& Lockman (1990) (see Sect. 5.4).

We computed hardness ratios as defined in Sect. 5.5 from the model spectra by adding up the computed count rates in the corresponding energy channels and minimized the function

$\Phi^{2}=\sum_{k=1}^{2} \frac{\left(H R k_{\mathrm{obs}}-H R k_{\mathrm{mod}}\right)^{2}}{\sigma(H R k)^{2}}$

with the observed and model hardness ratios $H R k_{\text {obs }}$ and $H R k_{\text {mod }}$, respectively, and $k=1,2$ (Schartel et al. 1996). The model spectra were computed with EXSAS. For each object $i$ the error of $\Gamma_{i}$ was estimated from $\Phi^{2}=\Phi_{\min }^{2}+1$ (one parameter problem, e.g. Lampton et al. 1976) as $\sigma_{i}=$ $\max (| \pm \Delta \Gamma|)$.

The histogram with the frequency distribution of the photon indices is displayed in Fig. 16. The mean photon index $\Gamma_{m}$ of the distribution was derived by means of a maximumlikelihood estimator. We assumed a Gaussian shape for the distribution with an intrinsic width $\sigma_{\Gamma}$. For $n$ data points the likelihood function is given by

$\mathcal{L}=\prod_{i=1}^{n} \frac{1}{\sqrt{2 \pi\left(\sigma_{\Gamma}^{2}+\sigma_{i}^{2}\right)}} \mathrm{e}^{-\frac{1}{2} \frac{\left(\Gamma_{m}-\Gamma_{i}\right)^{2}}{\left(\sigma_{\Gamma}^{2}+\sigma_{i}^{2}\right)}}$

(Maccacaro et al. 1988) with the individual photon indizes $\Gamma_{i}$ and their errors $\sigma_{i}$ for each object $i$ from Eq. (1). The test statistic $S=-2 \ln \mathcal{L}$ was minimized with respect to $\Gamma_{m}$ and $\sigma_{\Gamma}$. Errors for these parameters were obtained from $S=S_{\text {min }}+2.3$ (two parameter problem, e.g. Lampton et al. 1976). Results are summarized in Table 8. For the complete sample of 2215 AGN we thus obtained $\Gamma_{m}=2.48 \pm 0.03$ and a intrinsic width $\sigma_{\Gamma}=0.53 \pm 0.03$. For the radio-loud AGN we found $\Gamma_{m}=2.21 \pm 0.05$ and $\sigma_{\Gamma}=0.48 \pm 0.05$. For the radio-quiet sample the result was $\Gamma_{m}=2.56 \pm 0.03$ and $\sigma_{\Gamma}=0.50 \pm 0.05$. The results show that the X-ray continuum slope of radio-loud sources is significantly steeper than that of radio-quiet sources. The intrinsic widths of the distributions, however, are equal.

These findings are in very good agreement with the results of Schartel et al. (1996). They studied the power-law photon indizes of a sample of 102 bright quasars. For the soft energy band observed with ROSAT $(0.1-2.4 \mathrm{keV})$ they derived mean photon indizes of $\langle\Gamma\rangle_{\mathrm{rl}}=2.23 \pm 0.07$ and $\langle\Gamma\rangle_{\mathrm{rq}}=2.54 \pm 0.04$ for radio-loud and radio-quiet quasars, respectively. The slopes from ROSAT were found to be significantly steeper than those determined from observations in the harder energy range, e.g. with EINSTEIN (0.3-3.5 keV), EXOSAT (2-10 keV) and Ginga (2-20 keV). The slopes from ROSAT and EINSTEIN differ by 0.66 and 0.68 for radio-loud and radio-quiet quasars, respectively. These differences were explained by Schartel et al. as being due to the shape of the energy distribution. They suggested that a curved shape of the spectrum caused by an excess in the soft energy band can explain the steeper slope observed in soft range with ROSAT.
Table 8. Photon index for AGN power-law X-ray spectra.

\begin{tabular}{lll}
\hline \hline & $\Gamma_{m}$ & $\sigma_{\Gamma}$ \\
\hline all AGN & $2.48 \pm 0.03$ & $0.53 \pm 0.03$ \\
radio-loud & $2.21 \pm 0.05$ & $0.48 \pm 0.05$ \\
$R>10$ & & \\
radio-quiet & $2.56 \pm 0.03$ & $0.50 \pm 0.05$ \\
$R_{\lim } \leq 10$ & & \\
\hline
\end{tabular}

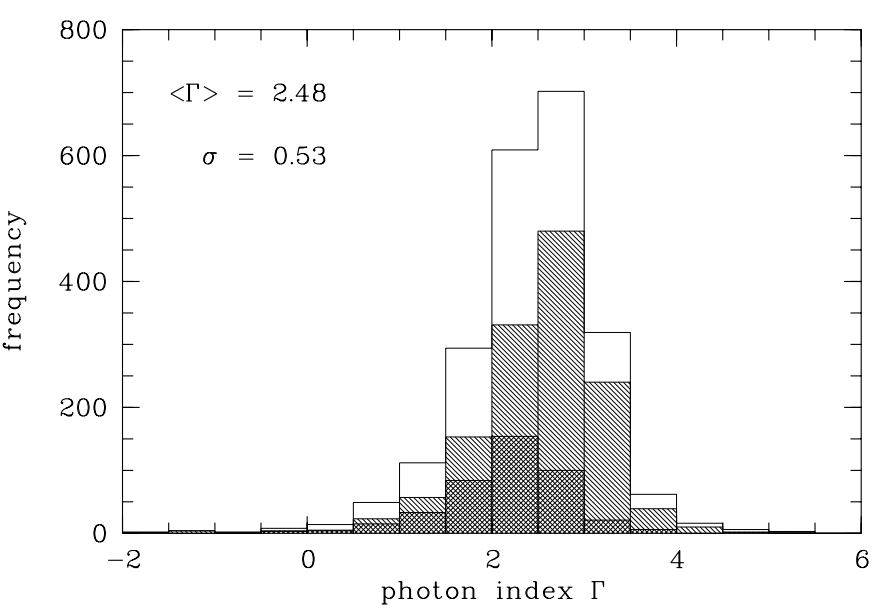

Fig. 16. Photon index $\Gamma$ of the 2215 AGN. The open histogram depicts the full sample. The mean value is $\Gamma=2.48 \pm 0.03$ and the intrinsic width of the distribution is $\sigma=0.53 \pm 0.03$. The left hashed and the double-hashed histograms represent the radio-quiet and radio-loud AGN, respectively.

\subsection{Continuum properties of stellar sources}

For coronal emitters of classes $\mathrm{F}-\mathrm{G} /$ bright, $\mathrm{K}$ and $\mathrm{M}$ star we calculated coronal temperatures using the same method as before for the calculation of the photon index for AGN. For the spectrum we assumed a one-temperature Raymond-Smith model. Model parameter was the coronal temperature, $T_{\text {cor }}$. Galactic $N_{\mathrm{H}}$ was kept fixed at $1 \times 10^{18} \mathrm{~cm}^{-2}$. Furthermore, solar abundances were assumed. The resulting temperatures are plotted as histograms in Fig. 17.

The frequency distribution for F-G/bright stars is bimodal with a minimum around $0.6 \mathrm{keV}$. It shows a dominant peak at $0.34 \mathrm{keV}$, i.e. $4.0 \times 10^{6} \mathrm{~K}$ with $73 \%$ of the stars in the two classes below $0.6 \mathrm{keV}$. A second peak appears at $1 \mathrm{keV}$, i.e. $11.6 \times 10^{6} \mathrm{~K}$ with the remaining $27 \%$ of stars above $0.6 \mathrm{keV}$. K and $\mathrm{M}$ stars have temperatures around $0.30 \mathrm{keV}$, i.e. $3.5 \times 10^{6} \mathrm{~K}$. There are also a few $\mathrm{K}$ and $\mathrm{M}$ stars in the high temperature range. The fraction of high temperature coronae is, however, smaller for later spectral types. For K and M stars $16 \%$ and $7 \%$, respectively, have temperatures above $0.6 \mathrm{keV}$.

The positional cross-correlation with the NVSS was also carried out for stellar counterparts. In 18 cases we found a radio source within the $90 \%$ radio error circle of $10^{\prime \prime}$ around the optical position. The majority of these stars are bright stars, 2 are $\mathrm{M}$ stars and 1 is a $\mathrm{K}$ star. 


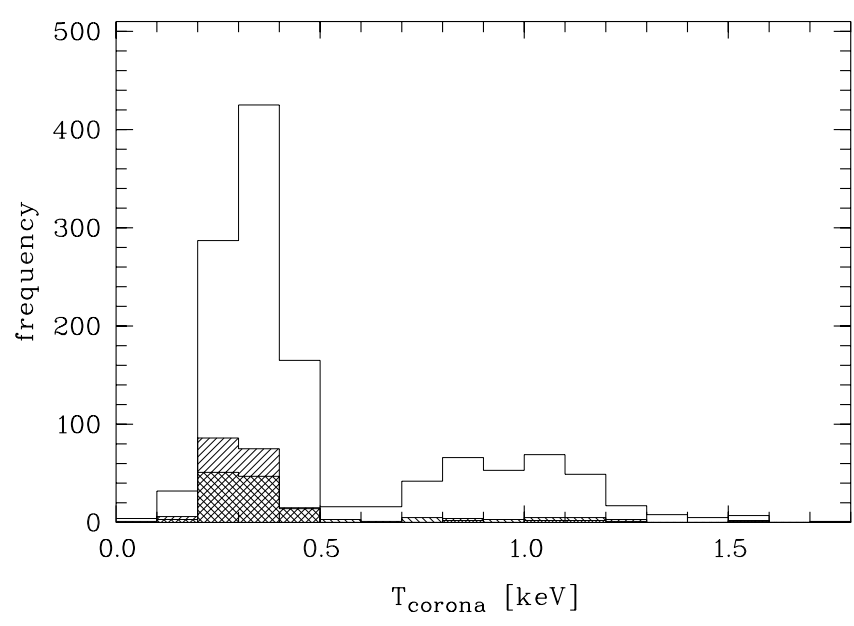

Fig. 17. Coronal temperatures of stellar sources of spectral types $M$ (right hashed), K (left hashed), and of F-G and bright stars (open histogram).

\section{8. $X$-ray $\log N-\log S$ distributions}

In Fig. $18 \log N-\log S$ distributions are shown for AGN, stars, and unidentified sources. In order to derive the slopes of the distributions we assumed a cumulative distribution function $N(>S)=k S^{-\alpha}$ and used the maximum-likelihood method described by Crawford et al. (1970) to estimate $\alpha$.

For the total AGN sample we obtained $\alpha=1.40 \pm 0.05$ for the flux range $2.5 \times 10^{-12} \leq S \leq 4.0 \times 10^{-11} \mathrm{erg} \mathrm{s}^{-1} \mathrm{~cm}^{-2}$ for which the distribution is linear. The slope for the radioquiet AGN is $\alpha=1.41 \pm 0.07$ and for the radio-loud AGN $\alpha=1.27 \pm 0.09$. Within the uncertainties the slopes are thus compatible with each other and with the Euclidean slope of $3 / 2$. Below $2.5 \times 10^{-12} \mathrm{erg} \mathrm{s}^{-1} \mathrm{~cm}^{-2}$ the slope becomes flatter possibly indicating incompleteness. For the LSW sample Krautter et al. (1999) derived a slope of $1.41 \pm 0.09$ which agrees well with our result. Both, the LSW and our HRC slope, appear flatter than the slope derived from the EMSS for which Della Ceca et al. (1992) obtained $\alpha=1.61 \pm 0.06$ for $S<10^{-11} \mathrm{erg} \mathrm{s}^{-1} \mathrm{~cm}^{-2}$. However, the differences are on a level of $\lesssim 2 \sigma$ only. Note that inclusion of all unidentified sources would only insignificantly steepen our slope of the total sample to $\alpha=1.43 \pm 0.05$. The $\log N-\log S$ distribution for unidentified sources is also shown in Fig. 18. The slope is $\alpha=1.62 \pm 0.12$, hence also compatible with the Euclidean slope of $3 / 2$.

For stellar sources we obtained $\alpha=1.19 \pm 0.03$ for the flux interval $4 \times 10^{-13} \leq S \leq 4 \times 10^{-11} \mathrm{erg} \mathrm{s}^{-1} \mathrm{~cm}^{-2}$. The slope is thus significantly flatter than Euclidean. The subgroup of coronal emitters (F-G/bright, $\mathrm{K}$, and $\mathrm{M}$ stars) yields a slightly yet insignificantly steeper slope of $\alpha=1.22 \pm 0.04$. Inclusion of all unidentified sources in the stellar group would steepen the slope to $\alpha=1.54 \pm 0.05$, i.e. compatible with the Euclidean slope.

\section{Summary}

We presented a catalogue of optical identifications of 5341 northern high-galactic latitude X-ray sources from

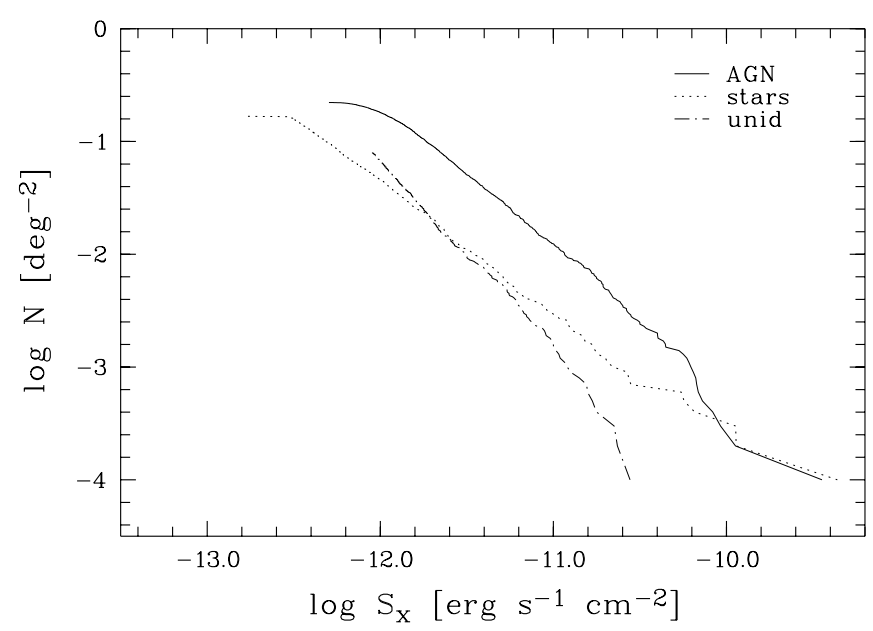

Fig. 18. $\log N-\log S$ distributions for AGN, stars, and unidentified sources.

the RASS-BSC. The catalogue contains $\sim 51 \%$ extragalactic sources and $\sim 31 \%$ galactic stellar X-ray emitters. The majority of extragalactic sources are active galactic nuclei (Sy, QSOs, BL Lacs) ( $\sim 1 \%$ of all sources). Stellar sources are mostly coronal emitters. In $3 \%$ of the sources no optical counterpart was visible on our blue direct plates. Another $15 \%$ of the sources remain unidentified.

In general, the source statistics of our catalogue is in good agreement with previous studies of smaller samples from the RASS. Some small differences in the frequency distribution of the individual object classes seem to be present, though.

A surprisingly large fraction of about $20 \%$ of AGN in the $\mathrm{HRC}$ is radio-loud. This is about twice as much as in the LBQS and in the EMSS. Our analysis of the X-ray spectra of AGN showed that radio-loud AGN have significantly harder X-ray continua than radio-quiet AGN.

The HRC is a valuable data base for the selection of complete samples of soft X-ray emitters for detailed follow-up studies.

Acknowledgements. This work was supported by DARA under grant Verbundforschung BMBF 50 OR 96060 . We would like to thank the referee Dr. Perlman for his constructive comments which helped to improve the paper. The Hamburg Quasar Survey (HQS) was supported by the Deutsche Forschungsgemeinschaft through grants $\operatorname{Re} 353 / 11-1,2,3$ and $\operatorname{Re} 353 / 22-1,2$, 3. The ROSAT project was supported by the Bundesministerium für Bildung und Wissenschaft and the Max-Planck-Gesellschaft. The Digitized Sky Survey was produced at the Space Telescope Science Institute under U.S. Government grant NAG W-2166.

\section{Appendix A: Sample spectra}

Sample spectra for the various spectral classes were extracted from the digital data base of the HQS prism plates. For the spectral classes of stars (F-M stars, CVs, white dwarfs) and AGN (including QSOs and BL Lacs) we selected objects with known characteristics from various catalogues. For each object we give the classification according to our object classes and in parentheses the catalogue classification and brightness. 


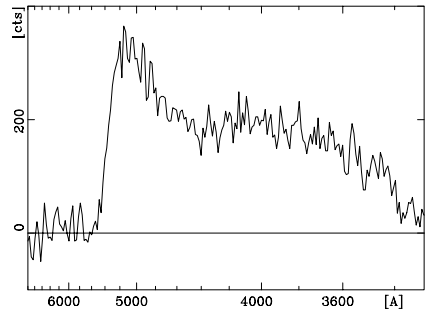

$\operatorname{AGN}(17.4, \mathrm{z}=0.78)$

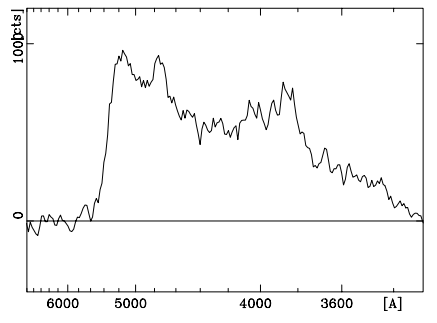

QSO $(15.9, \mathrm{z}=1.50)$

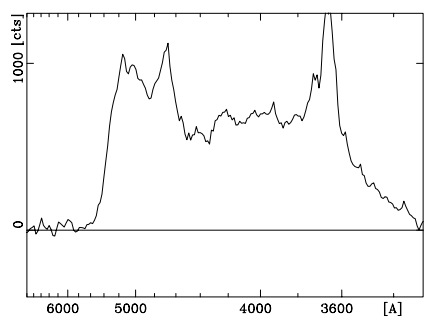

QSO $(\mathrm{B}=15.5, \mathrm{z}=2.04)$

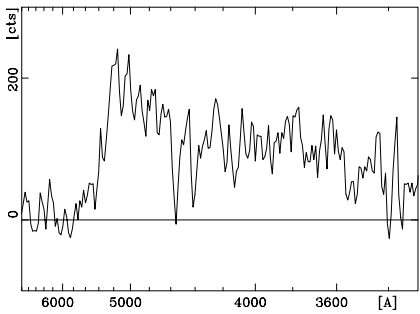

EBL-WK $(18.9, \mathrm{z}=1.00)$

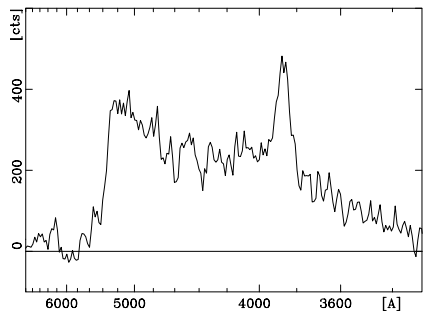

QSO $(17.1, z=1.52)$

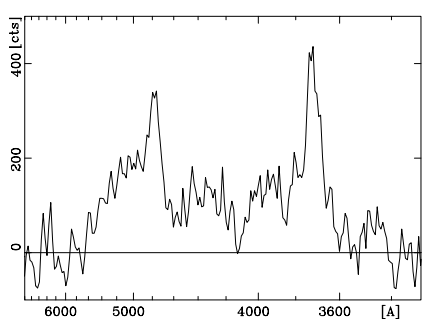

QSO $(18.2, \mathrm{z}=2.07)$

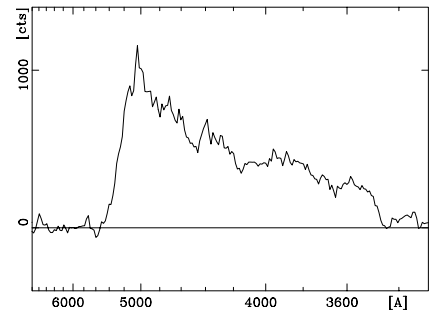

$\operatorname{AGN}(15.9, \mathrm{z}=1.32)$

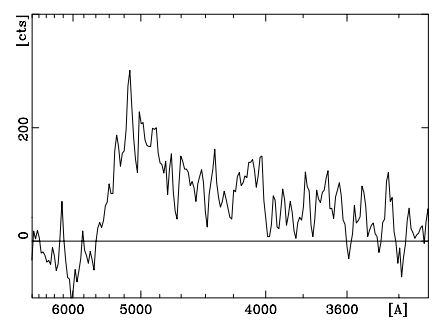

EBL-WK (18.5, z=1.66)

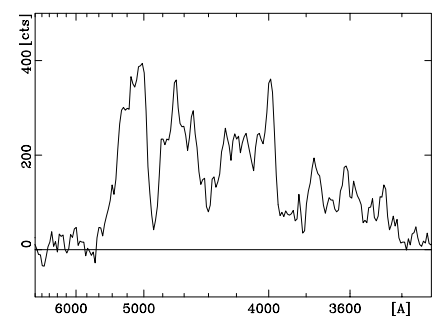

UNID $(18.9, \mathrm{z}=2.20)$

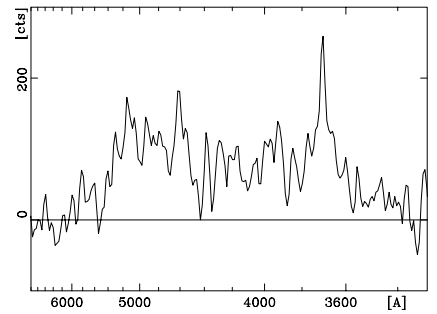

QSO $(19.1, \mathrm{z}=1.39)$

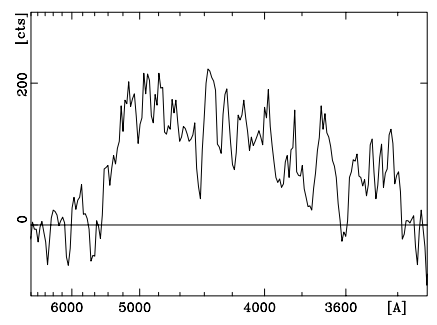

EBL-WK $(17.5, \mathrm{z}=1.83)$

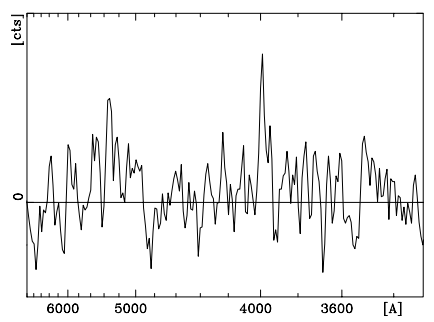

EBL-WK (19.4, z=2.24)

Fig. A.1. Sample spectra of QSOs with various redshifts and brightnesses.

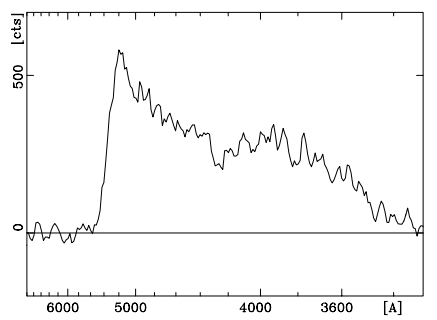

AGN (BL Lac, 17.0)

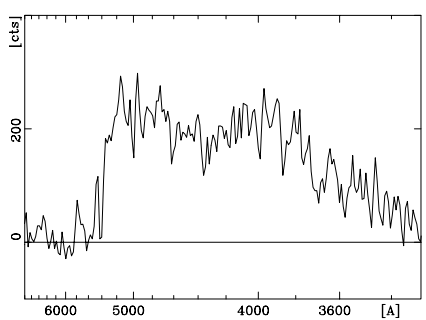

AGN (BL Lac, 18.0)

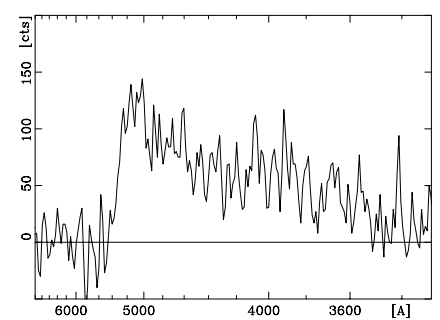

EBL-WK (BL Lac, 18.4)

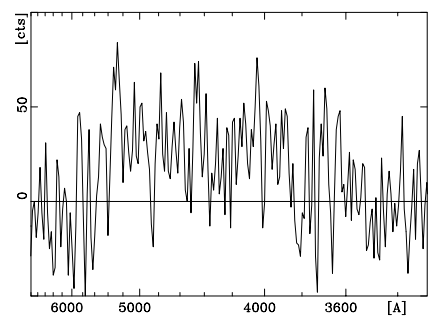

EBL-WL (BL Lac, 18.9)

Fig. A.2. Sample spectra of BL Lac objects with various brightnesses.

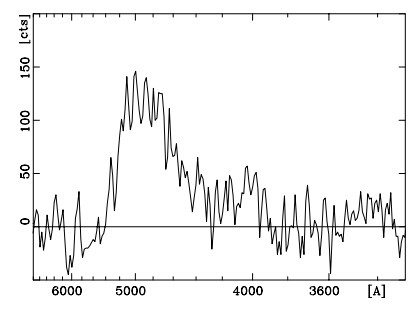

GAL $(\approx 17.5)$

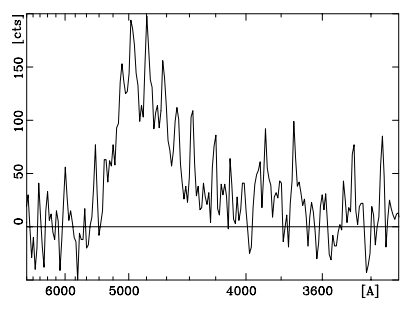

GAL $(\approx 17.5)$

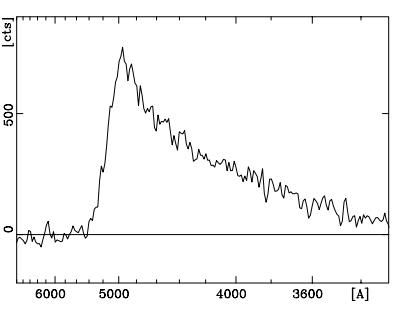

BLUE GAL $(\approx 17)$

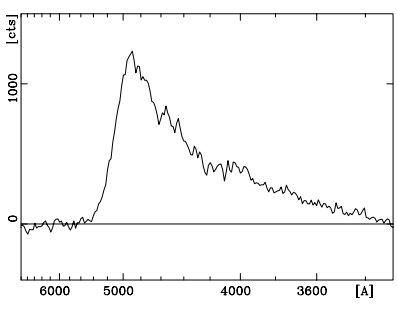

$\operatorname{BLUE~GAL~}(\mathrm{S} 0, \approx 17)$

Fig. A.3. Sample spectra of galaxies. 


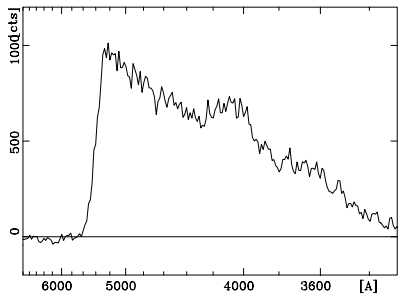

STAR-FG (F5V, 14.8)

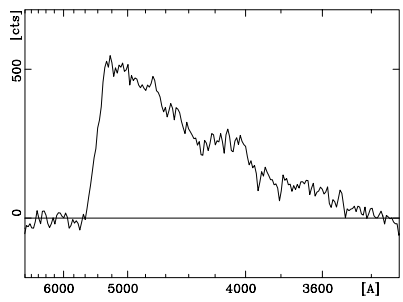

STAR-FG (G8V, 16.1)

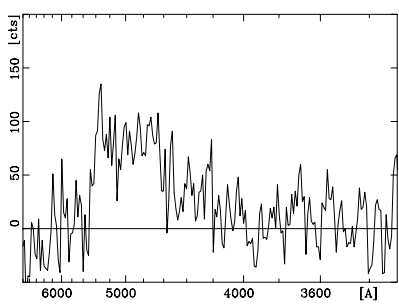

RED-WK (K3, 18.3)

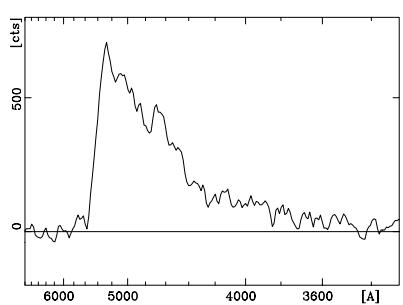

STAR-M (M3, 17.0)

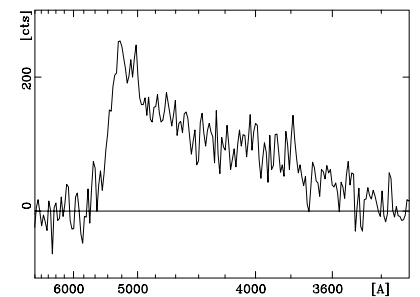

STAR-FG (F, 17.1)

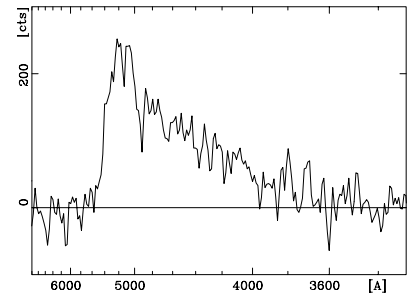

STAR-K (G, 17.4)

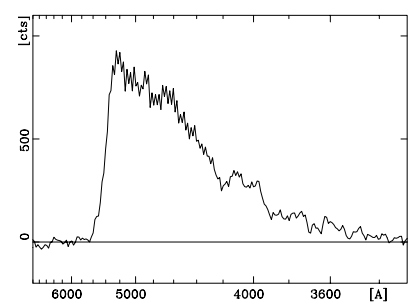

STAR-K (K7V, 15.1)

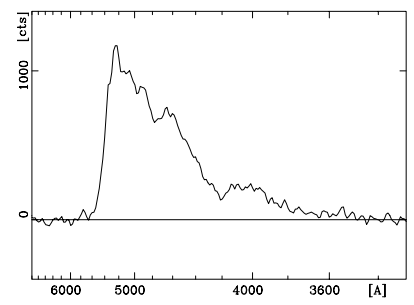

STAR-M (M4V, 15.3)

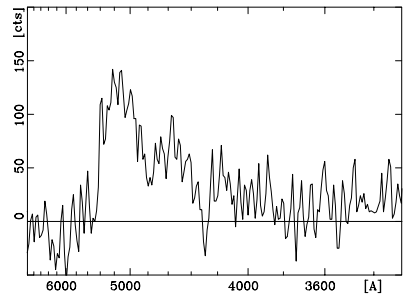

RED-WK (F, 17.3)

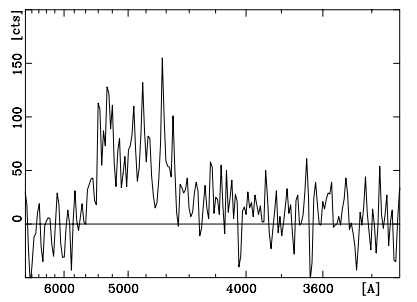

RED-WK (G, 18.3)

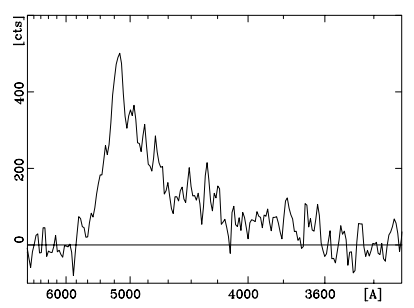

RED-WK (K, 16.8)

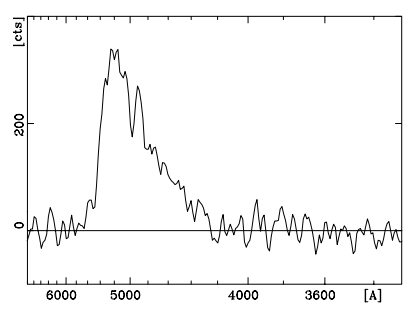

STAR-M (M7V, 16.1)

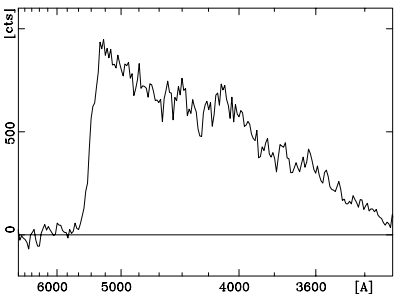

STAR-FG (G0, 15.0)

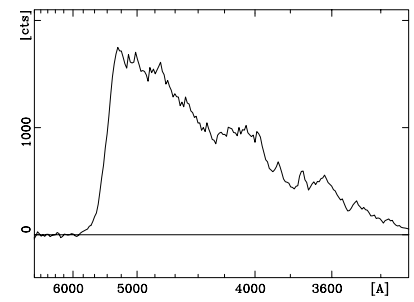

STAR-FG (K0, 14.2)

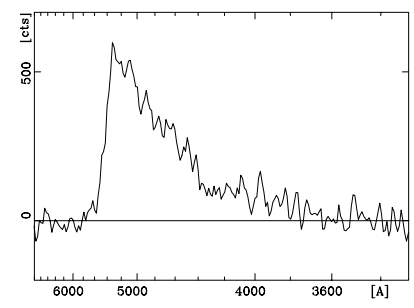

STAR-M (M2V, 16.8)

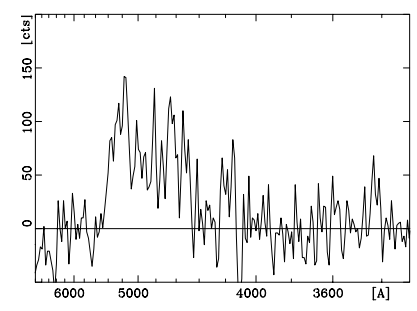

RED-WK (M, 18.7)

Fig. A.4. Sample spectra of F, G, K and M type stars.

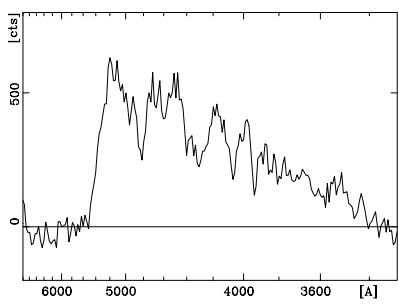

WD (DA5, 15.9)

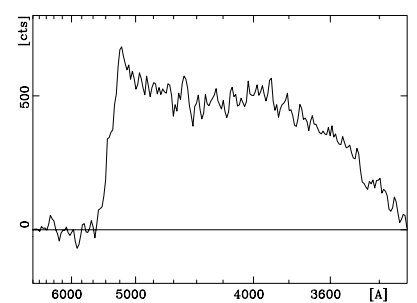

WD (DB, 16.2)

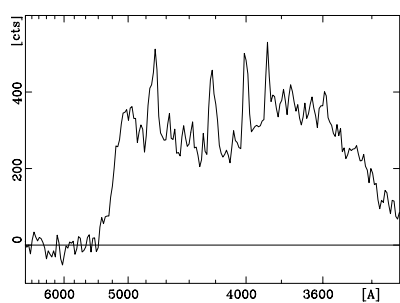

CV (Dwarf Nova, 16.8)

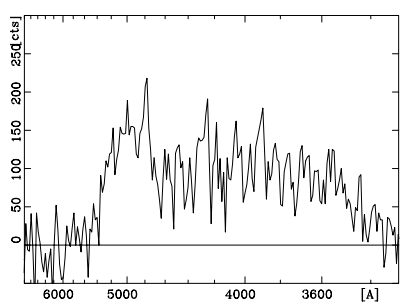

CV (Dwarf Nova, 18.2)

Fig. A.5. Sample spectra of white dwarfs and cataclysmic variables.

\section{References}

Appenzeller, I., Thiering, I., \& Zickgraf, F.-J., et al. 1998, ApJS, 117, 319

Aschenbach, B. 1988, Appl. Opt., 27, 1404

Bade, N., Engels, D., Voges, W., et al. 1998a, A\&AS, 127, 145 (Paper I)

Bade, N., Beckmann, V., Douglas, N. G., et al. 1998b, A\&A, 334, 459

Beckmann, V., Engels, D., Bade, N., \& Wucknitz, O. 2003, A\&A, 401, 927

Bischoff, O. B., \& Becker, R. H. 1997, AJ, 113, 2000

Brinkmann, W., Siebert, J., Feigelson, E. D., et al. 1997, A\&A, 323, 739

Caccianiga, A., Maccacaro, T., Wolter, A., Della Ceca, R., \& Gioia, I. 1999, ApJ, 513, 51

Condon, J. J., Cotton, W. D., Greisen, E. W., et al. 1998, AJ, 115, 1693

Crawford, D. F., Jauncey, D. L., \& Murdoch, H. S. 1970, ApJ, 162, 405 
Della Ceca, R., Maccacaro, T., Gioia, I. M., Wolter, A., \& Stocke, J. T. 1992, ApJ, 389, 491

Della Ceca, R., Zamorani, G., Maccacaro, T., et al. 1994, ApJ, 430, 533

Dickey, J. M., \& Lockman, F. J. 1990, ARA\&A, 28, 215

Engels, D., Cordis, L., \& Köhler, S. 1994, in Astronomy from widefield imaging, ed. H. T. MacGillivray, E. B. Thomson, B. M. Lasker, et al. (Dordrecht: Kluwer), IAU Symp., 161, 317

Engels, D., Hagen, H.-J., Cordis, L., et al. 1998, A\&AS, 128, 507

Fischer, J.-U., Hasinger, G., Schwope, A. D., et al. 1998, AN, 319, 347

Gioia, I. M., Maccacaro, T., Schild, R. E., et al. 1990, ApJS, 72, 567

Gregory, P. C., Scott, W. K., Douglas, K., \& Condon, J. J. 1996, ApJS, 103,427

Hagen, H.-J., Groote, D., Engels, D., \& Reimers, D. 1995, A\&AS, 111,195

Henry, J. P., Gioia, I. M., Mullis, C. R., et al. 2001, ApJ, 553, L109

Hooper, E. J., Impey, C. D., Foltz, C. B., \& Hewett, P. C. 1996, ApJ, 473,746

Kellermann, K. I., Sramek, R., Schmidt, M., Shaffer, D. B., \& Green, R. 1989, AJ, 98, 1195

Krautter, J., Zickgraf, F.-J., Appenzeller, I., et al. 1999, A\&A, 350, 743

La Franca, F., Gregorini, L., Cristiani, S., de Ruiter, H., \& Owen, F. 1994, AJ, 108, 1548

Lamla, E. 1982, in Landolt-Börnstein VI, 2b, ed. K. Schaifers, \& H. H. Voigt (Berlin: Springer)

Lampton, M., Margon, B., \& Bowyer, S. 1976, ApJ, 208, 177

Landt, H., Padovani, P., Perlman, E. S., et al. 2001, MNRAS, 323, 757

Laurent-Muehleisen, S. A., Kollgaard, R. I., Ryan, P. J., et al. 1997, A\&AS, 122, 235

Laurent-Muehleisen, S. A., Kollgaard, R. I., Ciardullo, R., et al. 1998, ApJS, 118, 127
Laurent-Muehleisen, S. A., Kollgaard, R. I., Feigelson, E. D., Brinkmann, W., \& Siebert, J. 1999, ApJ, 525, 127

Maccacaro, T., Gioia, I. M., Wolter, A., Zamorani, G., \& Stocke, J. T. 1988, ApJ, 326, 680

Mickaelian, A. M., Gonçalves, A. C., Véron-Cetty, M.-P., \& Véron, P. 2001, Astrophys., 44, 21

Morrison, R., \& McCammon, D. 1983, ApJ, 270, 119

Mukai, K. 1993, Legacy, 3, 21

Monet, D., Bird, A., Canzian, B., et al. 1998, The USNO-A2.0 Catalogue (Washington DC: U.S. Naval Observatory)

Padovani, P. 1993, MNRAS, 263, 461

Padovani, P., \& Giommi, P. 1995, ApJ, 444, 567

Perlman, E. S., Stocke, J. T., Schachter, J. F., et al. 1996, ApJS, 104, 251

Perlman, E. S., Padovani, P., Giommi, P., et al. 1998, ApJ, 115, 1253

Rector, T. A., Stocke, J. T., Perlman, E. S., Morris, S. L., \& Gioia, I. M. 2000, AJ, 120, 1626

Schartel, N., Walter, R., Fink, H. H., \& Trümper, J. 1996, A\&A, 307, 33

Schmidt, M. 1968, ApJ, 151,393

Stocke, J. T., Morris, S. L., Gioia, I. M., et al. 1991, ApJS, 76, 813

Schwope, A., Hasinger, G., Lehmann, I., et al. 2000, AN, 321, 1

Tananbaum, H., Avni, Y., Branduardi, G., et al. 1979, ApJ, 234, L9

Trümper, J. 1983, Adv. Space Res., 2, 4, 241

Urry, C. M., \& Padovani, P. 1995, PASP, 107, 803

Voges, W., Aschenbach, B., Boller, Th., et al. 1999, A\&A, 349, 389

Voges, W., Aschenbach, B., Boller, Th., et al. 2000, VizieR On-line Data Catalog: IX/29. Originally published in: Max-Planck-Institut fur extraterrestrische Physik (Garching)

Voges, W., Henry, J. P., Briel, U. G., et al. 2001, ApJ, 553, L119

Zickgraf, F.-J., Thiering, I., Krautter, J., et al. 1997, A\&AS, 123, 103

Zimmermann, H. U., Becker, W., Belloni, T., et al. 1994, MPE Rep., 257 